Copyright (C) 2005 IEEE. Reprinted from

IEEE Transactions on Geoscience and Remote Sensing, 2005; 43

(9):2058-2074

This material is posted here with permission of the IEEE. Such permission of the IEEE does not in any way imply IEEE endorsement of any of the University of Adelaide's products or services. Internal or personal use of this material is permitted. However, permission to reprint/republish this material for advertising or promotional purposes or for creating new collective works for resale or redistribution must be obtained from the IEEE by writing to pubs-permissions@ieee.org.

By choosing to view this document, you agree to all provisions of the copyright laws protecting it. 


\title{
Statistical Assessment of Eigenvector-Based Target Decomposition Theorems in Radar Polarimetry
}

\author{
Carlos López-Martínez, Member, IEEE, Eric Pottier, Member, IEEE, and Shane R. Cloude, Fellow, IEEE
}

\begin{abstract}
The performance of quantitative remote sensing based on multidimensional synthetic aperture radars (SARs), and polarimetric SAR systems in particular, depends strongly on a correct statistical characterization of the data, i.e., on a complete knowledge of the effects of the speckle noise. In this framework, the eigendecompostion of the covariance or coherency matrices and the associated $H / \underline{\alpha} / A$ decomposition have demonstrated the potential for quantitative estimation of physical parameters. In this paper, we present a detailed study of the statistics associated with this decomposition. This analysis requires the introduction of mathematical tools that are not well known in the remote sensing community. For this reason, we include a review section to present them. Using this work, we then present an expression for the probability density function of the sample eigenvalues of the covariance or coherency matrix. The availability of this expression allows a complete study of the separated sample eigenvalues, as well as, the entropy $H$ and the anisotropy $A$. As demonstrated, all these parameters must be considered as asymptotically nonbiased with respect to the number of looks. In order to reduce the biases for a small number of averaged samples, a novel estimator for the eigenvalues is proposed. The results of this work are analyzed by means of simulated and real airborne SAR data. This analysis permits us to determine in detail the effects of the number of averaged samples in the estimation of physical information in radar polarimetry.
\end{abstract}

Index Terms-Eigendecomposition, estimation theory, polarimetry, speckle, synthetic aperture radar (SAR), target decomposition theorems.

\section{INTRODUCTION}

A $\mathrm{T}$ present, there is increasing interest in the combination of multidimensional radar imagery along with microwave scattering models with the objective to retrieve quantitative physical information about the Earth's surface. In this context, radar polarimetry has largely demonstrated a leading role, thanks to its application in the framework of synthetic aperture radar (SAR) technology [1]. On the one hand, since these systems are active, their monitoring capabilities are independent of the weather conditions and of the day-night cycle. On the other hand, the coherent recording and processing of data makes it possible to retrieve Earth surface information with high spatial resolution. One of the most important consequences of this

Manuscript received November 29, 2004; revised April 28, 2005. This work was supported by the EU-RTN Network AMPER under Contract HPRH-CT2002-00205.

C. López-Martínez and E. Pottier are with the Universite Rennes 1, IETR, S.A.P.H.I.R. Team, 35042 Rennes Cedex, France (e-mail: carlos.lopez@ univ-rennes1.fr).

S. Cloude is with the School of Electrical and Electronic Engineering, University of Adelaide, Adelaide, SA 5005 Australia.

Digital Object Identifier 10.1109/TGRS.2005.853934 coherent nature is that SAR data are affected by speckle noise [2]-[4]. For one-dimensional (1-D) SAR systems, speckle noise has been completely characterized by a multiplicative noise model due to the exponential probability density function (pdf) of the SAR image intensity [2], [3]. In the case of multidimensional SAR systems, and for polarimetric SAR (PolSAR) systems in particular, the characterization and reduction of the speckle noise component has been an important area of research since it represents one of the key limitations of this technology [5]-[8]. Recently, it has been demonstrated that the speckle noise component for multidimensional SAR data, under a covariance matrix formulation, must be modeled through a combination of multiplicative and additive noise sources [9]. Therefore, it is important to determine in which way speckle noise affects the retrieval of physical information from multidimensional SAR data.

The bridge between radar measurements and the physical parameters of the scatterer under study can be established by the so-called target decomposition (TD) theorems [12], [13], [15]-[17]. Among the different approaches proposed in the literature, TD theorems based on the eigendecomposition of the coherency matrix are one of the most suitable tools to perform data interpretation in the study and characterization of natural scatterers [13], [18]. The extraction of physical information via the eigenvalues and eigenvectors, or the secondary parameters, i.e., the entropy $H$, the anisotropy $A$ and the $\alpha$ and $\beta$ angles, has been demonstrated by different authors. Some important applications of the coherency matrix eigendecomposition are unsupervised data classification [19], [20] and natural risks management [21], [22]. Another fruitful application is the extraction of quantitative surface parameters as proposed by Cloude [23], applied to the PolSAR data by Hajnsek et al. [24], [25], and extended by Allain et al. [26]. The same principle has been employed to solve inverse problems on vegetation remote sensing [27]. The main observation at this point is that speckle noise shall also affect the eigenvalues and eigenvectors associated with the eigendecomposition of the coherency, or covariance matrices. Consequently, a detailed study of the speckle noise effects over the eigendecomposition is necessary in order to understand the effects of this noise component on the quantitative estimation of the physical parameters of interest. When dealing with multidimensional SAR data, interest lies in the Hermitian, semidefinite positive coherency or covariance matrices. Recently, several studies have pointed in this direction [28]-[30]. But despite this, the problem has been largely considered in the mathematical community for symmetric, real, covariance matrices [31] and the case of complex Hermitian matrices did not arouse the same level of interest. 
This paper presents an in-depth study of the statistics characterizing the eigendecomposition of Hermitian, semipositive definite coherency or covariance matrices. The final objective is to assess the estimated physical information quantitatively and to determine the influence of the different processing parameters, as for instance: the number of averaged samples or looks, the number of data channels, etc. The next section introduces the basics of the statistical PolSAR data description. Section III presents a review of the results concerning the sample eigenvalues joint pdf for Hermitian matrices, which are extended in Section IV. The pdf of the sample eigenvalues is employed in Section V to characterize the sample eigenvalues, as well as the entropy and the anisotropy. Section VI presents some observations about the $\underline{\alpha}$ parameter. Section VII validates and studies the developed theory by means of simulated and real PolSAR data. Finally, Section VIII contains the conclusions of this study.

\section{MULTIDIMENSIONAL SAR DATA CHARACTERIZATION}

\section{A. Multidimensional SAR Data Description}

A PolSAR system measures the $2 \times 2$ complex scattering matrix $\mathbf{S}$. In order to better understand the physical information contained within the data, vectorization of the $\mathbf{S}$ matrix has been proposed in the literature [13], [32]. This vector-based formulation allows the extension of the ideas and concepts developed for PolSAR data to arbitrary multidimensional SAR systems, e.g., polarimetric SAR interferometry (PolInSAR) [33]. Thus, despite the fact that this paper is focused on the analysis of PolSAR data, the study presented in the following is performed from the perspective of a general multidimensional SAR system.

A multidimensional SAR system acquires $m$ complex SAR images $S_{i}$ for $i=1,2, \ldots, m$, represented by the target vector

$$
\mathbf{k}=\left[S_{1}, S_{2}, \ldots, S_{m}\right]^{T}
$$

where ${ }^{T}$ indicates transposition. In (1), the subindex indicates that the SAR images are acquired under some type of diversity. In this paper, interest is on polarization diversity, in which $\mathbf{k}$ is a four-dimensional vector in a general case, or a three-dimensional (3-D) vector for backscattering under the BSA (backscatter alignment) convention. For point scatterers, $\mathbf{k}$ is an $m$-dimensional deterministic vector. Nevertheless, remote sensing applications are primarily interested in the analysis of natural or distributed targets. Thus, $\mathbf{k}$ turns into an $m$-dimensional random variable [13] which can not be employed to characterize a distributed target without an assessment of the consequences of the speckle noise component [34]. For homogeneous data, under the Gaussian scattering assumption and on the basis of the central limit theorem, $\mathbf{k}$ is described by a zero-mean, multidimensional, complex Gaussian pdf [5], [6]

$$
p_{\mathbf{k}}(\mathbf{k})=\frac{1}{\pi^{m}|\mathbf{C}|} \exp \left(-\mathbf{k}^{H} \mathbf{C}^{-1} \mathbf{k}\right)
$$

where ${ }^{H}$ denotes transpose complex conjugation. The pdf in (2) is completely characterized by the $m \times m$, Hermitian, semipositive definite covariance matrix $\mathbf{C}$

$$
\begin{aligned}
\mathbf{C} & =E\left\{\mathbf{k k}^{H}\right\} \\
& =\left[\begin{array}{cccc}
E\left\{S_{1} S_{1}^{H}\right\} & E\left\{S_{1} S_{2}^{H}\right\} & \cdots & E\left\{S_{1} S_{m}^{H}\right\} \\
E\left\{S_{2} S_{1}^{H}\right\} & E\left\{S_{2} S_{2}^{H}\right\} & \cdots & E\left\{S_{2} S_{m}^{H}\right\} \\
\vdots & \vdots & \ddots & \vdots \\
E\left\{S_{m} S_{1}^{H}\right\} & E\left\{S_{m} S_{2}^{H}\right\} & \cdots & E\left\{S_{m} S_{m}^{H}\right\}
\end{array}\right] .
\end{aligned}
$$

where $E\{x\}$ is the expectation operator. In the literature, departures from the model given by (2) have been reported, especially at high spatial resolutions or longer wavelengths. These deviations are mainly due to the presence of data texture [35], [36]. Nevertheless, (2) is considered in the following as its simplicity allows the analytical study of the eigendecomposition of $\mathbf{C}$.

Due to the speckle noise, $\mathbf{C}$ must be estimated from the data in (1). Under the assumption that $S_{i}$, for $i=1,2, \ldots, m$, in (1) are ergodic in mean and in correlation, the ensemble average in (3) can be substituted by a spatial averaging. Additionally, this spatial averaging makes sense only if the processes $S_{i}$ and $S_{i} S_{j}^{*}$ for $i, j=1,2, \ldots, m$ are wide-sense stationary in mean. Therefore, $\mathbf{C}$ can be estimated by means of

$$
\mathbf{Z}_{n}=\frac{1}{n} \sum_{i=1}^{n} \mathbf{k}_{i} \mathbf{k}_{i}^{H} .
$$

The matrix $\mathbf{Z}_{n}$, which corresponds to the maximum-likelihood estimator (MLE) of C [37], receives the name of sample covariance matrix. In (4), the vectors $\mathbf{k}_{i}$ for $i=1,2, \ldots, n$ correspond to the target vectors of every one of the $n$ averaged samples. The statistics of $\mathbf{Z}_{n}$ are completely characterized by the Wishart distribution [5], [6], [38]

$$
p \mathbf{Z}_{n}\left(\mathbf{Z}_{n}\right)=\frac{n^{m n}\left|\mathbf{Z}_{n}\right|^{n-m}}{|\mathbf{C}|^{n} \tilde{\Gamma}_{m}(n)} \operatorname{etr}\left(-n \mathbf{C}^{-1} \mathbf{Z}_{n}\right)
$$

where etr( $\mathbf{X})$ is the exponential of the trace of the matrix $\mathbf{X}, n \geq m$, and the multivariate gamma function is defined as follows:

$$
\tilde{\Gamma}_{m}(n)=\pi^{m(m-1) / 2} \prod_{i=1}^{m} \Gamma(n-i+1) .
$$

In (6), $\Gamma(\mathrm{x})$ is the gamma function.

\section{B. Eigendecomposition}

In PolSAR, with the objective to better understand the information contained in the data from a physical point of view, a different vectorization of the scattering matrix $\mathbf{S}$ has been proposed. Whereas (1) corresponds to a vectorization in the so-called lexicographic matrix basis, a vectorization based on the Pauli matrix basis, giving rise to the target vector $\mathbf{k}_{p}$, has been considered [13]. From $\mathbf{k}_{p}$ the coherency matrix is defined as

$$
\mathbf{T}=E\left\{\mathbf{k}_{p} \mathbf{k}_{p}^{H}\right\}
$$


An important class of TD theorems is based on the eigendecomposition of the matrix $\mathbf{T}$ [13]. The eigenvectors can be considered as a set of orthonormal scattering mechanisms, whereas the corresponding eigenvalue is interpreted as the scatterer power associated with the eigenvector. It is evident that the presence of the speckle noise affects the eigendecomposition of T. Nevertheless, the characterization of speckle noise for $\mathbf{T}$ results difficult as its different entries do not present the same mathematical structure. This drawback can be overcome by considering the equivalent matrix $\mathbf{C}$, since all its entries correspond to the Hermitian products of the components of (1) [9]. Since $\mathbf{C}$ and $\mathbf{T}$ are related by a unitary similarity transformation, they present the same eigenvalues but not the same eigenvectors. As a result, the eigenvalue decomposition of $\mathbf{C}$ is considered in the following.

Let $\mathbf{C}$ be a $m \times m$ Hermitian, semipositive define matrix. Its eigendecomposition is

$$
\boldsymbol{\Sigma}=\mathbf{Q}^{\prime H} \mathbf{C Q}^{\prime}
$$

where

$$
\boldsymbol{\Sigma}=\left[\begin{array}{cccc}
l_{1} & 0 & \cdots & 0 \\
0 & l_{2} & \cdots & 0 \\
\vdots & \vdots & \ddots & \vdots \\
0 & 0 & \cdots & l_{m}
\end{array}\right]
$$

and $l_{i}$ for $i=1,2, \ldots, m$ denote the eigenvalues of $\mathbf{C}$, called in the following the true eigenvalues. The $m \times m$, complex, unitary matrix $\mathbf{Q}$ ' contains the eigenvectors of $\mathbf{C}$ as its columns. A general unitary matrix is specified by $m^{2}$ independent parameters. Nevertheless, the matrix Q' is defined by $m(m-1)$ independent parameters since the eigenvectors can only be specified up to a phase component. This phase term corresponds to the absolute phase of an absolute target vector. As a consequence, Q' is defined up to $m$ phase components. The eigenvalues $l_{i}$ for $i=1,2, \ldots, m$ are considered as the set of primary parameters of the eigendecomposition. Additionally, two secondary parameters have been defined in the frame of PolSAR: the entropy $H$ and the anisotropy $A$ [13], [19]. For backscattering, i.e., $m=3, H$ is defined as

$$
H=-\sum_{i=1}^{3} p_{i} \log _{3}\left(p_{i}\right) \quad p_{i}=\frac{l_{i}}{\sum_{j=1}^{3} l_{j}}
$$

whereas the anisotropy $A$ is defined as

$$
A=\frac{l_{2}-l_{3}}{l_{2}+l_{3}} \text {. }
$$

The $H / \underline{\alpha} / A$ decomposition [13] contains also the parameter $\underline{\alpha}$, which is derived from the eigenvectors of $\mathbf{T}$, which can be modeled by

$$
\begin{aligned}
& \mathbf{e}_{i}=\left[\begin{array}{lll}
\cos \alpha_{i} e^{j \phi_{i}^{1}} & \sin \alpha_{i} \cos \beta_{i} e^{j \phi_{i}^{2}} \quad \sin \alpha_{i} \cos \beta_{i} e^{\phi_{i}^{3}}
\end{array}\right]^{T}, \\
& i=1,2,3 \text {. }
\end{aligned}
$$

These vectors present five independent parameters, represented in terms of five angles, which can be divided into an absolute phase plus four additional independent parameters. From (12), considering also (10), $\underline{\alpha}$ is defined as

$$
\underline{\alpha}=\sum_{i=1}^{3} p_{i} \alpha_{i} .
$$

Despite separately $\mathbf{e}_{i}$ for $i=1,2,3$ present five independent parameters, together they form an orthonormal basis. This condition imposes additional restrictions in the number of independent parameters of $\mathbf{e}_{i}$, for $i=1,2,3$, resulting in $\mathbf{Q}$ ' to be defined by $m(m-1)$ independent parameters, i.e., six independent parameters for PolSAR in backscattering [14].

Since $\mathbf{C}$ is estimated by means of the sample covariance matrix $\mathbf{Z}_{n}$ as a consequence of the speckle noise, the eigendecomposition of $\mathbf{C}$ is estimated via the eigendecomposition of $\mathbf{Z}_{n}$. The eigendecomposition of $\mathbf{Z}_{n}$ is defined as

$$
\boldsymbol{\Xi}=\mathbf{Q}^{H} \mathbf{Z}_{n} \mathbf{Q}
$$

where

$$
\boldsymbol{\Xi}=\left[\begin{array}{cccc}
\lambda_{1} & 0 & \cdots & 0 \\
0 & \lambda_{2} & \cdots & 0 \\
\vdots & \vdots & \ddots & \vdots \\
0 & 0 & \cdots & \lambda_{m}
\end{array}\right]
$$

On the one hand, $\mathbf{Q}$ contains the eigenvectors of the sample covariance matrix $\mathbf{Z}_{n}$. On the other hand, the matrix $\boldsymbol{\Xi}$ contains $\lambda_{i}$ for $i=1,2, \ldots, m$ which in the following shall be named the sample eigenvalues. Similarly, the entropy (10), the anisotropy (11), and the $\underline{\alpha}$ angle (13) can be defined for $\mathbf{Z}_{n}$.

Since the matrix $\mathbf{Z}_{n}$ is characterized by the Wishart pdf (5), this distribution shall also determine the properties of its eigendecomposition. Consequently, it is important to determine the statistical properties of the eigenvalues and eigenvectors of $\mathbf{Z}_{n}$ and also, to establish the relation with the eigendecomposition of $\mathbf{C}$. The final result of this process is that, since the eigendecomposition can be employed to determine quantitatively certain physical properties of the scatterer under consideration, it shall be possible to determine the final effects of speckle noise over these physical quantities. The following study shall focus exclusively on the statistical characterization of the eigenvalues of the decomposition (14).

\section{SAMPle EIgEnValues PDF: A Review}

This section presents a review about the results concerning the joint pdf of the sample eigenvalues of $m \times m$ Hermitian, semipositive definite covariance matrices. The reason to give this survey is that, despite the fact that problem has already been considered for real, symmetric matrices [31], the complex case has received little attention, and results are scattered in the literature.

As observed in (14), the eigendecomposition of $\mathbf{Z}_{n}$ factorizes it into two matrices: $\boldsymbol{\Xi}$, which contains the $m$ sample eigenvalues and $\mathbf{Q}$, which contains $m(m-1)$ independent parameters. Consequently, the process to derive the marginal distribution of the sample eigenvalues, denoted as $p_{\Xi}(\boldsymbol{\Xi})$, must be divided into two steps. First, the transformation (14), along with the determinant of its Jacobian, must be introduced into the Wishart distribution (5). Second, the $m(m-1)$ parameters of $\mathrm{Q}$, i.e., the parameters which determine the eigenvectors must be integrated in order to derive $p_{\Xi}(\boldsymbol{\Xi})$. Since the eigenvectors are integrated, this process prevents the statistics of $\underline{\alpha}$ (13) from being studied. 
Equation (14) represents a multidimensional transformation process. The direct calculation of the Jacobian's determinant can be extremely complicated and tedious. The alternative is to obtain the Jacobian's determinant, by means of the exterior product of differential forms [39]-[41]. This approach is based on the observation that the determinant of a matrix behaves as the exterior product, since both are skew-symmetric, i.e., a permutation of two variables changes the sign of the product. The exterior product is also called wedge product, and it is denoted by the symbol $\wedge$. The concept of exterior algebra, as the algebra of the exterior product, was introduced by Herman G. Grassmann in 1844 in his extension theory [42]. In exterior algebras, ordinary vectors $\delta$ receive the name of 1 -forms, upon which the exterior product acts with the following basic properties:

- $\delta \wedge \delta=0$

- $(a \delta \wedge \eta)=a(\delta \wedge \eta)$

- $\delta \wedge \eta=-\eta \wedge \delta$, skew-symmetric property.

where $\eta$ is also a 1 -form and $a$ is a constant. Elie Cartan extended the exterior product to the study of the exterior product of differential forms under the observation that the product of differential 1-forms $d x d y$ behaves as $d x \wedge d y$. Let $V$ be an $m$-dimensional Euclidean space defined by the independent coordinates $x_{i}$, for $i=1,2, \ldots, m$. Thus, a differential volume is defined as

$$
\bigwedge_{i=1}^{m} d x_{i}=d x_{1} \wedge \cdots \wedge d x_{m}
$$

The other important operation which applies to differential forms is the exterior derivative, which extends the idea of derivative. The exterior derivative, denoted by $d$, takes each $p$-form $\omega$ to a $(p+1)$-form $d \omega$ by considering that for each $\omega, d(d \omega)=0$. The interest on the exterior product of independent differential forms lies in the fact that the Jacobian's determinant of a given transformation can be derived as the exterior product of differential forms. Given a transformation of coordinates $\phi: \mathbb{R}^{m} \rightarrow \mathbb{R}^{m}$, where $x_{i}$, for $i=1,2, \ldots, m$, refer to the coordinates of the domain of $\phi$ and $y_{i}$, for $i=1,2, \ldots, m$, denote the coordinates of the range, then

$$
\bigwedge_{i=1}^{m} d y_{i}=\left|\frac{\partial y_{i}}{\partial x_{j}}\right| \bigwedge_{i=1}^{m} d x_{i}
$$

In (17), the term $\left|\partial y_{i} / \partial x_{j}\right|$ can be clearly identified as the Jacobian's determinant of the corresponding transformation $\phi$ : $\mathbb{R}^{m} \rightarrow \mathbb{R}^{m}$. Given a matrix $\mathbf{A}$, the exterior product of the independent differential components $d a_{i j}$ is denoted by $(d \mathbf{A})$, called the volume element. For instance, for a general $m \times n$ matrix $\mathbf{A}(d \mathbf{A})=\Lambda_{i=1}^{m} \Lambda_{j=1}^{n} d a_{i j}$, whereas for a $m \times m$, symmetric matrix, with $(1 / 2) m(m+1)$ independent parameters, $(d \mathbf{A})=\Lambda_{1 \leq i \leq j \leq m} d a_{i j}$. Until this point, only real differential forms have been considered. Given a $z$ complex, $(d z)=$ $d \Re\{z\} \wedge d \Im\{z\}$. Equally, for a complex matrix $\mathbf{A},(d \mathbf{A})=$ $(d \Re\{\mathbf{A}\}) \wedge(d \Im\{\mathbf{A}\})$.

Given the eigendecomposition of the matrix $\mathbf{Z}_{n}$ (14), the exterior product of its independent differential forms is [43]

$$
\left(d \mathbf{Z}_{n}\right)=\prod_{i<j}^{m}\left(\lambda_{i}-\lambda_{j}\right)^{2}\left(\mathbf{Q}^{H} d \mathbf{Q}\right)(d \boldsymbol{\Xi})
$$

where

$$
\infty>\lambda_{1} \geq \lambda_{2} \geq \cdots \geq \lambda_{m} \geq 0
$$

Considering (17), it is possible to identify the Jacobian's determinant of the eigendecomposition in (18). Consequently, one can find the joint pdf of the eigenvalues and eigenvectors of $\mathbf{Z}_{n}$ by introducing (14) and (18) into (5). After minor manipulations, one gets the differential form of the pdf

$$
\begin{aligned}
p_{\mathbf{Z}_{n}}\left(\mathbf{Z}_{n}\right)\left(d \mathbf{Z}_{n}\right)= & \frac{n^{m n} \prod_{i=1}^{m} \lambda_{i}^{n-m} \prod_{i<j}^{m}\left(\lambda_{i}-\lambda_{j}\right)^{2}}{\tilde{\Gamma}_{m}(n) \prod_{i=1}^{m} l_{i}^{n}} \\
& \times \operatorname{etr}\left(-n \mathbf{C}^{-1} \mathbf{Q} \boldsymbol{\Xi} \mathbf{Q}^{H}\right)\left(\mathbf{Q}^{H} d \mathbf{Q}\right)(d \boldsymbol{\Xi}) .
\end{aligned}
$$

\section{A. Sample Eigenvalues PDF: Integral Expression}

In order to derive the marginal distribution of the sample eigenvalues $p_{\Xi}(\boldsymbol{\Xi})$, it is necessary to integrate the dependence on the $m \times m$ unitary matrix $\mathbf{Q}$ in (20). Since $\mathbf{Q} \in U(m)$, i.e., the unitary group of matrices, the integration process must be undertaken within $U(m)$. Equation (20) depends on $\mathbf{Q}$ through $\operatorname{etr}\left(-n \mathbf{C}^{-1} \mathbf{Q} \Xi \mathbf{Q}^{H}\right)$, and on the exterior product of differential forms $\left(\mathbf{Q}^{H} d \mathbf{Q}\right)$. Therefore, the nature of this second component needs to be considered.

Let $\mathbf{q}_{i}$, for $i=1,2, \ldots, m$, be the complex orthonormal columns of $\mathbf{Q} \in U(m)$. Then, the differential exterior product

$$
\left(\mathbf{Q}^{H} d \mathbf{Q}\right)=\stackrel{i=1}{\Lambda}_{i=1}^{m} \mathbf{q}_{j}^{H} \mathbf{q}_{i}
$$

defines a unique measure over the group $U(m)$, which often receives the name of Haar measure [44], [45]. Equation (21) is of extreme importance as it consists of the differential volume with respect to which the integration over $U(m)$ has to be defined [46]. An important quantity is the so-called volume of the complex unitary group

$$
\begin{aligned}
\operatorname{Vol}[U(m)] & =\int_{U(m)}\left(\mathbf{Q}^{H} d \mathbf{Q}\right) \\
& =\frac{2^{m} \pi^{m^{2}}}{\pi^{m(m-1) / 2} \prod_{i=1}^{m} \Gamma(m-i+1)} .
\end{aligned}
$$

Occasionally, instead of (22), it is more convenient to define a differential volume with respect to which the integration over all the space is equal to 1. Doing so, the space of interest is transformed into a probability space, in which probabilities can be measured. The normalized differential volume is defined as

$$
(d \mathbf{Q})=\frac{\left(\mathbf{Q}^{H} d \mathbf{Q}\right)}{\operatorname{Vol}[U(m)]} .
$$

Equation (23) is now considered in (20) in order to integrate the dependence on the eigenvectors in the matrix Q. Additionally, (20) must be divided by $(2 \pi)^{m}$ in order to account for the absolute phase of the eigenvectors. Thus

$$
\begin{gathered}
p_{\boldsymbol{\Xi}}(\boldsymbol{\Xi})=\frac{\pi^{m(m-1)} n^{m n} \prod_{i=1}^{m} \lambda_{i}^{n-m} \prod_{i<j}^{m}\left(\lambda_{i}-\lambda_{j}\right)^{2}}{\tilde{\Gamma}_{m}(n) \tilde{\Gamma}_{m}(m) \prod_{i=1}^{m} l_{i}^{n}} \\
\times \int_{U(m)} \operatorname{etr}\left(-n \mathbf{C}^{-1} \mathbf{Q} \boldsymbol{\Xi} \mathbf{Q}^{H}\right)(d \mathbf{Q}) .
\end{gathered}
$$


Equation (24) represents the joint pdf of the sample eigenvalues of the matrix $\mathbf{Z}_{n}$, but as can be observed, it has to be considered as an integral expression due to the integral over $U(m)$.

\section{B. Sample Eigenvalues PDF: Infinite Series Expression}

The integral term in (24) can not be solved by a term-byterm integration. Nevertheless, it can be obtained on the basis of the group representation theory [47] and the zonal polynomials theory [49]. In the next section, these theories are briefly presented, where interest shall be placed on those concepts and properties which allow solving the integral expression in (24).

Formally, a group representation of a group $G$ is defined as a group action of $G$ on a given vector space $V$ by means of invertible maps. The interest here lies on the particular representation of the group $G L(m, \mathbb{C})$, i.e., the group of all $m \times m$, invertible matrices $\mathbf{L}$ over $\mathbb{C}$. In this case, the representation considers the space $V_{k}$ of polynomials $p(\mathbf{R})$ which are homogeneous of degree $k$ in the entries of $m \times m$, Hermitian, positive definite matrices, denoted by $\mathbf{R}$. Nevertheless, concern is on the consequences that the representation of $G L(m, \mathbb{C})$ has over the space $V_{k}$, since the representation itself induces a decomposition of $V_{k}$ into the direct sum of $m$ 1-D invariant subspaces $V_{\kappa}$ [48]

$$
V_{k}=\bigoplus_{\kappa} V_{\kappa}
$$

where $\kappa=\left(k_{1}, k_{2}, \ldots, k_{m}\right)$ runs through all the partitions of $k$ into at most $m$ parts; that is, $k_{1} \geq k_{2} \geq \cdots \geq k_{m} \geq 0$ are integers and $k_{1}+k_{2}+\cdots+k_{m}=k$. A polynomial $\tilde{C}_{\kappa}(\mathbf{R})$, which generates every one of the 1-D subspaces $V_{\kappa}$, is called the complex zonal polynomial of the representation [49]. The properties of these polynomials permit to solve the integral in (24). Thus, a small description of them is presented in the following.

A complex zonal polynomial $\tilde{C}_{\kappa}(\mathbf{R})$ consists of a symmetric, homogeneous polynomial of degree $k$ in the entries of the matrix $\mathbf{R}$, which generates the subspace $V_{\kappa}$. From the definition of the representation of $G L(m, \mathbb{C})$ itself, the space $V_{\kappa}$ is invariant under the unitary group of matrices $U(m)$ [48]. This invariability of $V_{\kappa}$ implies that the complex zonal polynomials present the following property [49]:

$$
\tilde{C}_{\kappa}\left(\mathbf{Q R Q} \mathbf{Q}^{H}\right)=\tilde{C}_{\kappa}(\mathbf{R}) \quad \mathbf{Q} \in U(m)
$$

i.e., complex zonal polynomials depend on the matrix $\mathbf{R}$ only through its eigenvalues. In opposition with respect to real zonal polynomials (defined in the frame of $G L(m, \mathbb{R})$ which are defined hierarchically [31], complex zonal polynomials present an analytical expression as they consist, except for a normalization constant, of the so-called Schur polynomials [50]. The main property of interest of complex zonal polynomials in the frame of the distribution presented in (24) is the so-called splitting property. Following James [51] and Takemura [49], let A and $\mathbf{B}$ be $m \times m$, Hermitian, positive definite matrices and $\mathbf{Q} \in U(m)$. Then

$$
\int_{U(m)} \tilde{C}_{\kappa}\left(\mathbf{A Q B Q}{ }^{H}\right)(d \mathbf{Q})=\frac{\tilde{C}_{\kappa}(\mathbf{A}) \tilde{C}_{\kappa}(\mathbf{B})}{\tilde{C}_{\kappa}\left(\mathbf{I}_{m \times m}\right)}
$$

where $\mathbf{I}_{m \times m}$ is the $m \times m$ identity matrix.

Going back to (24), one can observe, first, that its integral term contains the trace of the matrix $-n \mathbf{C}^{-1} \mathbf{Q} \boldsymbol{\Xi} \mathbf{Q}^{H}$. On the one hand, the polynomial $(\operatorname{tr} \mathbf{R})^{k}$ can be easily identified as a member of the space $V_{k}$. On the other hand, if one considers the factorization in (25), $(\operatorname{tr} \mathbf{R})^{k}$ can be decomposed as follows [49], [51]:

$$
(\operatorname{tr} \mathbf{R})^{k}=\sum_{\kappa} \tilde{C}_{\kappa}(\mathbf{R})
$$

where the sum goes over all the partitions $\kappa$ of $k$. The integral term in (24), depends on the trace of $-n \mathbf{C}^{-1} \mathbf{Q} \Xi \mathbf{Q}^{H}$ through the exponential function. Considering the polynomial expansion of the exponential function

$$
\operatorname{etr}(\mathbf{R})=\sum_{k=0}^{\infty} \frac{(\operatorname{tr} \mathbf{R})^{k}}{k !}=\sum_{k=0}^{\infty} \sum_{\kappa} \frac{\tilde{C}_{\kappa}(\mathbf{R})}{k !} .
$$

Considering (27) and (29) into (24) [51]

$$
\begin{array}{r}
p_{\boldsymbol{\Xi}}(\boldsymbol{\Xi})=\frac{\pi^{m(m-1)} n^{m n} \prod_{i=1}^{m} \lambda_{i}^{n-m} \prod_{i<j}^{m}\left(\lambda_{i}-\lambda_{j}\right)^{2}}{\tilde{\Gamma}_{m}(n) \tilde{\Gamma}_{m}(m) \prod_{i=1}^{m} l_{i}^{n}} \\
\times \sum_{k=0}^{\infty} \sum_{\kappa} \frac{\tilde{C}_{\kappa}\left(-n \boldsymbol{\Sigma}^{-1}\right) \tilde{C}_{\kappa}(\boldsymbol{\Xi})}{k ! \tilde{C}_{\kappa}\left(\mathbf{I}_{m \times m}\right)} .
\end{array}
$$

The infinite series in (30) represents the complex hypergeometric function of double matrix argument [51], [52]

$$
{ }_{0} \tilde{F}_{0}\left(-n \boldsymbol{\Sigma}^{-1}, \boldsymbol{\Xi}\right)=\sum_{k=0}^{\infty} \sum_{\kappa} \frac{\tilde{C}_{\kappa}\left(-n \boldsymbol{\Sigma}^{-1}\right) \tilde{C}_{\kappa}(\boldsymbol{\Xi})}{k ! \tilde{C}_{\kappa}\left(\mathbf{I}_{m \times m}\right)} .
$$

Despite the integral in (24) has been solved, (30) is expressed in terms of an infinite series. The series in (31) presents very complicated convergence properties, making necessary the evaluation of a high number of terms in order to have a reliable value of the series [53].

\section{SAMPLE EIgENVALUES PDF: DeterminANT EXPRESSION}

Equation (30) represents the joint sample eigenvalues pdf. Nevertheless, for practical purposes a simplification of (31) is necessary. The answer to this question can be found within soliton theory [54] and more specifically, on the concept of $\tau$ functions (tau functions) [55], [56]. The interest on this type of functions is on the fact that they permit us to obtain an analytical expression for the infinite series (31).

A soliton is defined as a stable and isolated, i.e., solitary, traveling nonlinear wave solution for a set of equations, or hierarchy of differential equations, that obeys a superposition-like principle, i.e., solitons passing through one another emerge unmodified. A $\tau$ function consists of a sort of potential which gives rise to these hierarchies of differential equations [57]. A $\tau$ function can be represented as $\tau=\tau\left(p, \mathbf{t}, \mathbf{t}^{*}\right)$ [55], [56], i.e., this function depends on a discrete variable $p$ and on two semiinfinite sets of variables $\mathbf{t}$ and $\mathbf{t}^{*}$ named higher times. In its more general form, a tau function presents the form of a double series over partitions in Schur functions [50], [58]. Nevertheless, interest is on the special class of $\tau$ functions called $\tau$ functions of hypergeometric type and matrix argument. The first specialization is to consider $\tau$ functions of hypergeometric type, in which the double series over partitions is simplified to a single series [59], as in (31). The second particularization is to introduce a change of variables into the $\tau$ functions through which $\mathbf{t}$ and $\mathbf{t}^{*}$ are transformed into the so-called Hirota-Miwa variables [49], 
denoted as $\mathbf{x}=\left[x_{1}, x_{2}, \ldots\right]^{T}$ and $\mathbf{y}=\left[y_{1}, y_{2}, \ldots\right]^{T}$. The interest on this second set of variables is that $\tau$ functions are symmetric functions with respect to them, and, hence, they can be considered as the eigenvalues of the Hermitian matrices $\mathbf{X}$ and $\mathbf{Y}$, respectively [57]. Consequently, the $\tau$ function of hypergeometric type and matrix argument can be written as [57]

$$
\tau_{r}(p, \mathbf{X}, \mathbf{Y})=\sum_{k=0}^{\infty} \sum_{\kappa} r_{\kappa}(p) s_{\kappa}(\mathbf{t}(\mathbf{x})) s_{\kappa}\left(\mathbf{t}^{*}(\mathbf{y})\right)
$$

where $s_{\kappa}(\mathbf{x})$ denote the Schur functions and $r_{\kappa}(\rho)$ is a finite function depending on the integer $p$ [59]. If one compares (32) with (31), it can be observed that both expressions are formally equal. The main characteristic of $\tau$ functions is that they present an analytical expression which permits its evaluation without the necessity to calculate the infinite series [59]. After selecting the function $r_{\kappa}(\rho)$ to be a rational function, and considering $\mathbf{X}=$ $\left[x_{1}, x_{2}, \ldots, x_{m}\right]^{T}$ and $\mathbf{y}=\left[y_{1}, y_{2}, \ldots, y_{m}\right]^{T}$ being the eigenvalues of the $m \times m$ Hermitian $\mathbf{X}$ and $\mathbf{Y}$ matrices, respectively

$$
{ }_{0} \tilde{F}_{0}(\mathbf{X}, \mathbf{Y})=c_{m}(m) \frac{\left.\left|\exp \left(x_{i} y_{j}\right)\right|\right|_{i, j=1} ^{m}}{\Delta(\mathbf{x}) \Delta(\mathbf{y})}
$$

where

$$
c_{m}(m)=\prod_{k=1}^{m-1} k^{m-k}
$$

and $\Delta(\mathbf{x})$ and $\Delta(\mathbf{y})$ represent the Vandermonde determinants of $\mathbf{x}=\left(x_{1}, x_{2}, \ldots, x_{m}\right)$ and $\mathbf{y}=\left(y_{1}, y_{2}, \ldots, y_{m}\right)$ respectively, defined as

$$
\Delta(\mathbf{x})=\prod_{i<j}\left(x_{i}-x_{j}\right) .
$$

In (33), the term $\left.\left|\exp \left(x_{i} y_{j}\right)\right|\right|_{i, j=1} ^{m}$ is a determinant whose elements are the exponential function of the product of the eigenvalues $x_{i} y_{j}$, where the subindices $i$ and $j$ correspond to he indices of the rows and columns of the matrix itself.

In order to derive the final expression of the joint sample eigenvalues pdf (33) must be considered in (30). Consequently, after some minor manipulations

$$
\begin{aligned}
& p_{\boldsymbol{\Xi}}(\boldsymbol{\Xi})=\frac{\pi^{m(m-1)} n^{\frac{m}{2}(2 n-m+1)}}{\tilde{\Gamma}_{m}(m) \tilde{\Gamma}_{m}(n)} \prod_{k=1}^{m-1} k^{m-k} \\
& \times\left.\frac{\prod_{i=1}^{m} \lambda_{i}^{n-m} \prod_{i<j}^{m}\left(\lambda_{i}-\lambda_{j}\right)}{\prod_{i=1}^{m} l_{i}^{n} \prod_{i<j}^{m}\left(l_{j}^{-1}-l_{i}^{-1}\right)}\left|\exp \left(-n \frac{\lambda_{j}}{l_{i}}\right)\right|\right|_{i, j=1} ^{m} .
\end{aligned}
$$

\section{A. Sample Eigenvalues PDF: Simplification and Analysis of Dependences}

The joint sample eigenvalues pdf given in (36) can be compacted as follows. On the one hand, the constant

$$
\begin{aligned}
& K\left(m, n, l_{1}, \ldots, l_{m}\right) \\
& \quad=\frac{\pi^{m(m-1)} n^{\frac{m}{2}(2 n-m+1)}}{\tilde{\Gamma}_{m}(m) \tilde{\Gamma}_{m}(n)} \frac{\prod_{k=1}^{m-1} k^{m-k}}{\prod_{i=1}^{m} l_{i}^{n} \prod_{i<j}^{m}\left(l_{j}^{-1}-l_{i}^{-1}\right)}
\end{aligned}
$$

is considered. On the other hand, the expression of a $m \times m$ matrix determinant as a sum over the symmetric group, denoted as $S_{m}$, is also taken into consideration to simplify (36). Hence, the determinant component in (36) can be considered as follows:

$$
\left.\left|\exp \left(-n \frac{\lambda_{i}}{l_{j}}\right)\right|\right|_{i, j=1} ^{m}=\sum_{\pi \in S_{m}} \operatorname{sgn}(\pi) \prod_{i=1}^{m} \exp \left(-n \frac{\lambda_{i}}{l_{\pi_{i}}}\right)
$$

where $\pi$, here, denotes a permutation of $\{1,2, \ldots, m\}$, i.e., an element of the symmetric group of degree $m$, i.e., $S_{m}$. Also, $\pi_{i}$, for $i=1,2, \ldots, m$, consist of each particular element of the partition $\pi$. The $\operatorname{symbol} \operatorname{sgn}(\pi)$ is the parity or the permutation $\pi$, which equals +1 or -1 according to whether $\pi$ is an even or odd permutation. Introducing (37) and (38) into (36)

$$
\begin{array}{r}
p_{\boldsymbol{\Xi}}(\boldsymbol{\Xi})=K\left(m, n, l_{1}, \ldots, l_{m}\right) \prod_{i=1}^{m} \lambda_{i}^{n-m} \prod_{i<j}^{m}\left(\lambda_{i}-\lambda_{j}\right) \\
\times \sum_{\pi \in S_{m}} \operatorname{sgn}(\pi) \prod_{i=1}^{m} \exp \left(-n \frac{\lambda_{i}}{l_{\pi_{i}}}\right) .
\end{array}
$$

It is worth to notice that all the sum terms of (39) present the same qualitative structure, differing only on the permutation of the true eigenvalues.

From (39), it can be observed that the joint sample eigenvalues pdf depends on the following:

- number of acquired SAR images $m$, i.e., the covariance matrix dimension;

- number of averaged samples, or looks, in order to derive the sample covariance matrix (4) $n$;

- value of the true eigenvalues $l_{i}$ for $i=1,2, \ldots, m$, which have to be considered as sorted, i.e., $\infty>l_{1} \geq l_{2} \geq \cdots \geq$ $l_{m} \geq 0$.

Additionally, (39) presents two limitations: it is only valid for $n \geq m$, as given by (5) and the sample eigenvalues need to be considered as sorted (19).

\section{SAMPLe EIgenVAlues STUdy}

This section focuses on the statistical analysis of the sample eigenvalues, the entropy $H$ and the anisotropy $A$, on the basis of (39). From this equation, it can be clearly observed that $p_{\Xi}(\boldsymbol{\Xi})$ can not be separated, in any case, as the product of the distributions of every particular sample eigenvalue $\lambda_{i}$, for $i=$ $1,2, \ldots, m$, that is, the sample eigenvalues can not be considered independent. As a consequence, in order to study the statistical behavior of a particular eigenvalue $\lambda_{j}$, the remaining eigenvalues $\lambda_{i}$, for $i=1,2, \ldots, m$ and $i \neq j$, must be integrated in (39), under the condition $\infty>\lambda_{1} \geq \lambda_{2} \geq \cdots \geq \lambda_{m} \geq 0$. The analytical solution of this integration is extremely complex. Thus, this study shall be conducted in two different directions. First, a general statistical analysis of the sample eigenvalues, in which $n \geq m$, shall be detailed. Due to the complexity of $p_{\Xi}(\boldsymbol{\Xi})$, this study is performed through numerical integration methods. In a second step, (39) is analyzed asymptotically with respect to the number of averaged samples $n$.

The statistical analysis of the sample eigenvalues has been performed in its most general form in the previous sections of 
this paper. Nevertheless, since this paper is especially focused PolSAR, the previous analysis is considered for PolSAR data in the backscattering case under the BSA convention, in which $m=3$.

\section{A. Case of Study: $n \geq m$}

An analytical analysis of the particular sample eigenvalues in its more general form, based on (39), is not feasible, since the resulting expressions for the distributions of the sample eigenvalues can only be given in an integral form. This analysis is even complex in the simple case in which $m$ is fixed to a value equal to 3 . A feasible possibility to analyze the sample eigenvalues is to consider numerical integration methods to obtain the distribution of a particular eigenvalue, together with its mean and variance values from (39). The main drawback of this approach is that all the parameters in (39) need be specified, making difficult the extraction of general conclusions since a complete exploration of the parameters space would be arduous.

For the case of analysis, PolSAR data in the backscattering case are considered. Hence, the true eigenvalues are fixed to

$$
\left\{l_{1}, l_{2}, l_{3}\right\}=\{3,2,1\} .
$$

The value of $n$ is not fixed, since its effect on the different quantities wants to be determined. Finally, the different numerical integration processes have to be performed according to the region of integration defined by $\infty>\lambda_{1} \geq \lambda_{2} \geq \lambda_{3} \geq 0$. Under these conditions, Fig. 1 presents the evolution of the distributions $p_{\lambda_{i}}\left(\lambda_{i}\right)$, for $i=1,2,3$, as a function of the number of averaged samples employed to construct $\mathbf{Z}_{n}$, ranging from 3 to 64 samples. As observed, the number of averaged samples has a clear effect on the distributions of the sample eigenvalues. In addition, the evolution of these differs among the different eigenvalues. The key values concerning the sample eigenvalues are their mean and variance values. The mean value will show whether the sample eigenvalue estimates correctly the corresponding true eigenvalue or not, whereas the variance contains the uncertainty of this estimation. The distributions presented in Fig. 1 have been employed to derive the mean and the variance values of the sample eigenvalues. Fig. 2 presents the evolution of the mean values (dotted curves) as a function of the number of averaged samples. The values of the true eigenvalues (40) have been also included. As it can be deduced, the sample eigenvalues $\lambda_{i}$, for $i=1,2, \ldots, m$, can be considered as asymptotically nonbiased estimators of the true eigenvalues $l_{i}$, for $i=1,2, \ldots, m$. In addition, Fig. 3 presents the variance value of the three sample eigenvalues. This figure clearly shows, that the variances decrease with the number of looks, but also that the variance depends on the value of the corresponding eigenvalue. The eigenvalues are also employed to derive the entropy $H$ (10) and the anisotropy $A$ (11) parameters. The corresponding mean and variance values are presented by Figs. 4 (dotted curves) and 5, respectively. As observed in Fig. 4, the biases of the eigenvalues are also translated to $H$ and $A$. Hence, the entropy and anisotropy derived from the sample eigenvalues are also asymptotically nonbiased with respect to the true values. The variances of these two parameters, as observed in Fig. 5, decrease with the

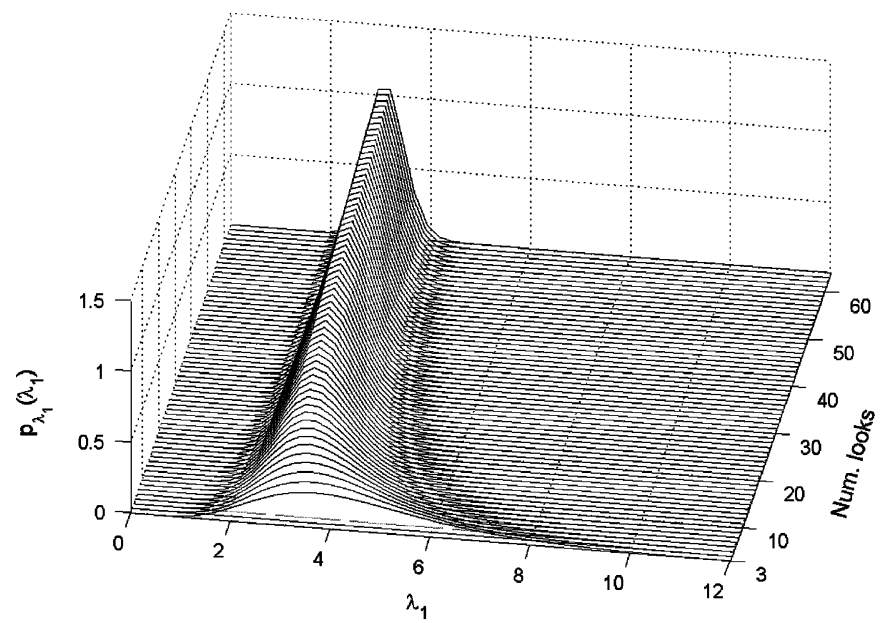

(a)

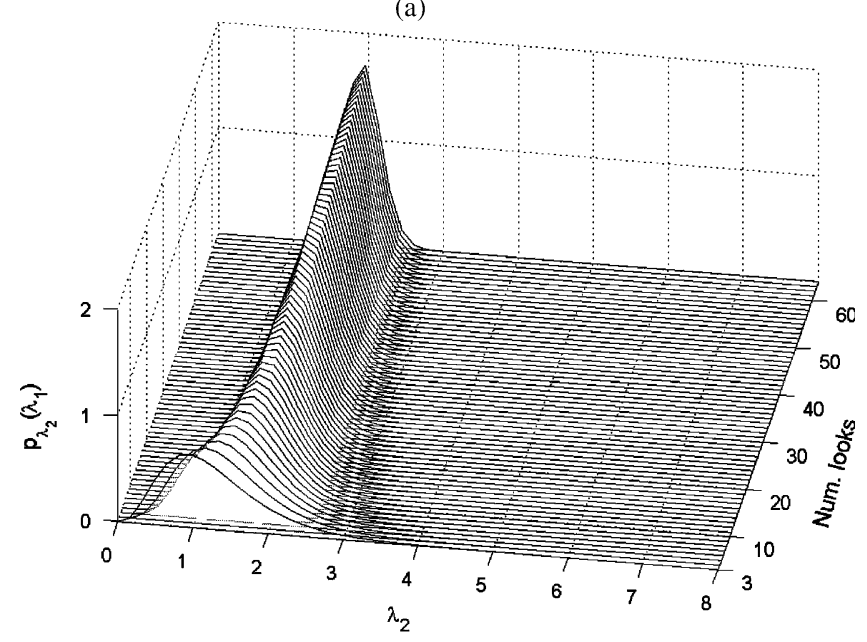

(b)

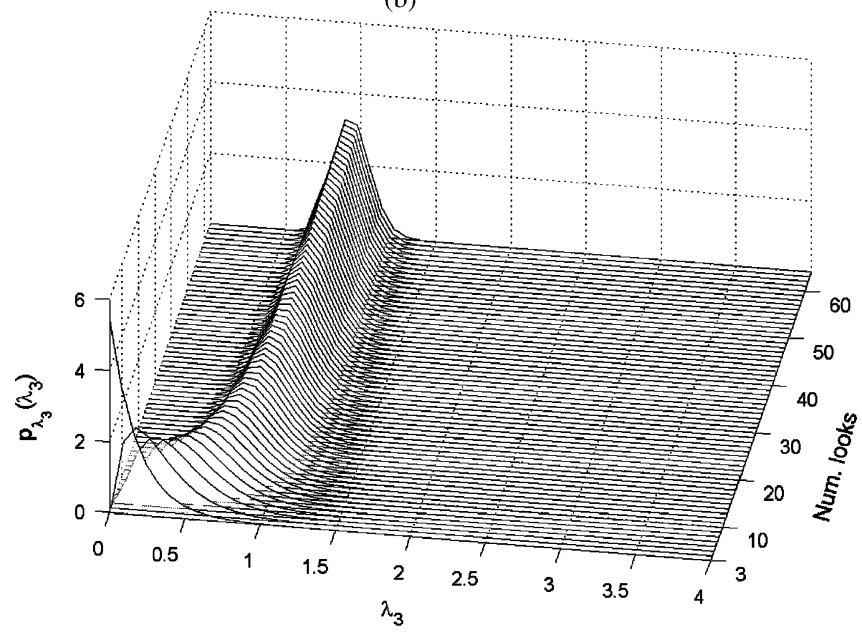

(c)

Fig. 1. Sample eigenvalues distributions for $n=3, \ldots, 64$ and $\left\{l_{1}, l_{2}, l_{3}\right\}=$ $\{3,2,1\}$. (a) $p_{\lambda_{1}}\left(\lambda_{1}\right)$. (b) $p_{\lambda_{2}}\left(\lambda_{2}\right)$. (c) $p_{\lambda_{3}}\left(\lambda_{3}\right)$.

number of looks. Fig. 5 also shows that the anisotropy is noisier than the entropy.

As demonstrated, the sample eigenvalues present a decreasing bias with respect to the corresponding true values, which can not be determined analytically due to the complexity associated with (39). 


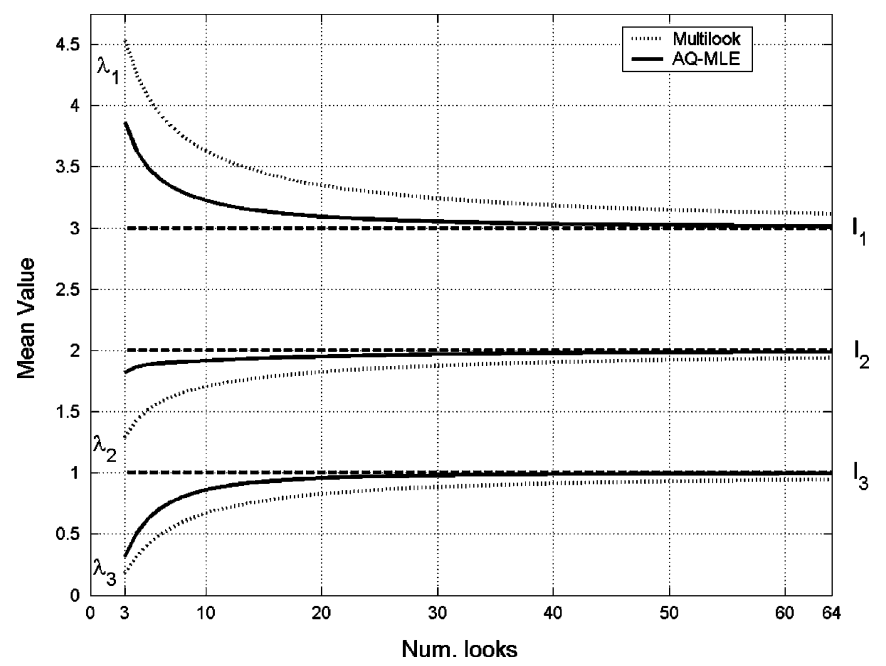

Fig. 2. Sample eigenvalues and AQ-MLE estimators of the eigenvalues $\left\{l_{1}, l_{2}, l_{3}\right\}=\{3,2,1\}$.

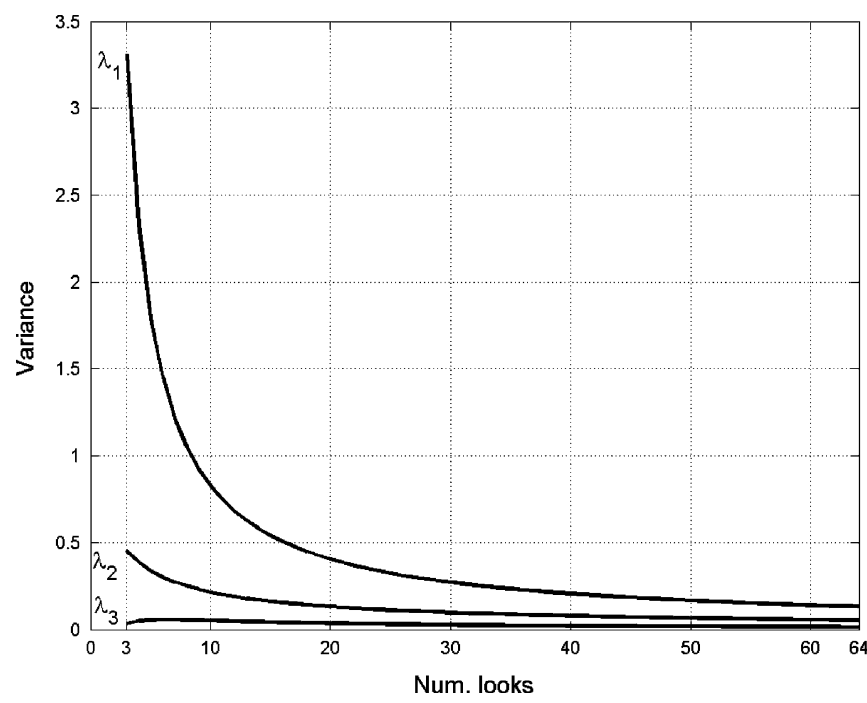

Fig. 3. Sample eigenvalues variance values for $n=3, \ldots, 64$ and $\left\{l_{1}, l_{2}, l_{3}\right\}=\{3,2,1\}$.

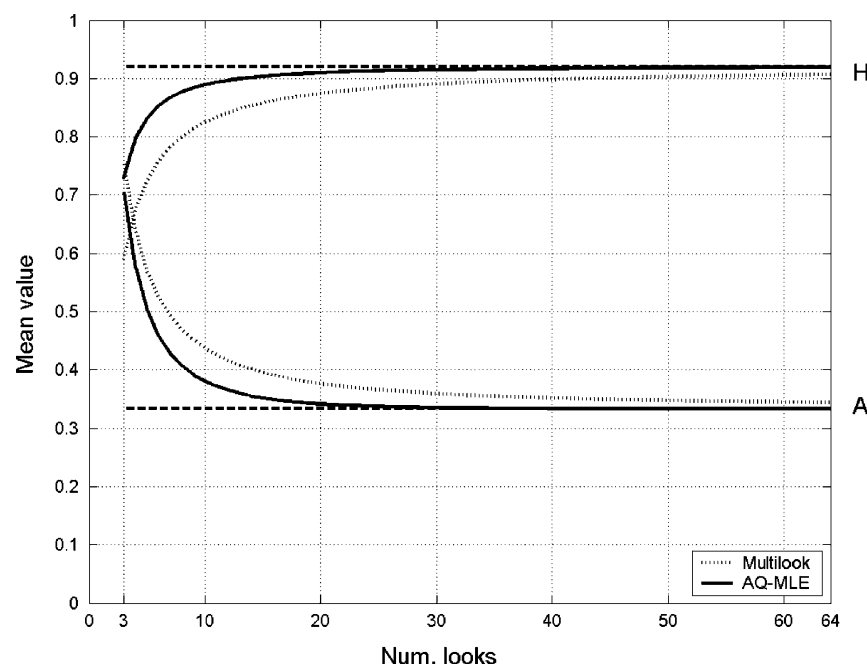

Fig. 4. Sample values and AQ-MLE estimators of the entropy $(\mathrm{H})$ and the anisotropy (A) $\left\{l_{1}, l_{2}, l_{3}\right\}=\{3,2,1\}$.

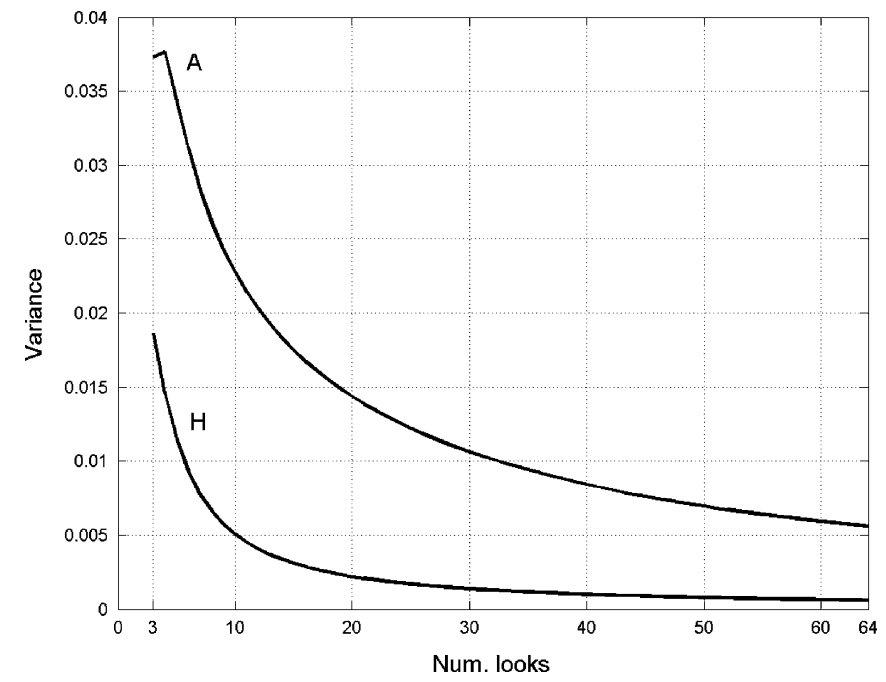

Fig. 5. Entropy and anisotropy variance values for $n=3, \ldots, 64$ and $\left\{l_{1}=\right.$ $\left.3, l_{2}=2, l_{3}=1\right\}$.

\section{B. Asymptotic Study/MLE Approach}

As concluded previously, the sample eigenvalues $\lambda_{i}$, for $i=$ $1,2, \ldots, m$, present a decreasing bias with respect to the true eigenvalues $l_{i}$, for $i=1,2, \ldots, m$. Consequently, these biases need to be characterized analytically with the objective to correct them. Since the joint distribution of the sample eigenvalues is available, (39), the maximum-likelihood estimator (MLE) approach could be explored. Nevertheless, this approach is not viable since the maximization of the log-likelihood function of (39) involves the maximization of a determinant. This maximization presents such a complex process to find the MLE of the true eigenvalues that it can not be considered for practical purposes.

1) Eigenvalues Asymptotic MLE (A-MLE): In order to derive an alternative expression for the MLE, (39) needs to be simplified. As given in (39), $p_{\Xi}(\boldsymbol{\Xi})$ consists of a sum over $S_{m}$, by introducing a permutation of the true eigenvalues $l_{i}$, for $i=1,2, \ldots, m$, within the exponential function. A given permutation of the eigenvalues different from the natural ordering $\left\{l_{1}, l_{2}, \ldots, l_{m}\right\}$, is characterized by the fact that the true eigenvalues do not follow the restriction $\infty>l_{1} \geq l_{2} \geq \cdots \geq l_{m} \geq 0$. In (39) it must be considered that $\infty>\lambda_{1} \geq \lambda_{2} \geq \cdots \geq \lambda_{m} \geq 0, \infty>l_{1} \geq l_{2} \geq \cdots \geq l_{m} \geq 0$ and the fact that the permutation affects only the exponential term. Let

$$
\begin{array}{r}
\exp \left(-n\left(\frac{\lambda_{1}}{l_{\pi_{1}}}+\cdots+\frac{\lambda_{i}}{l_{\pi_{i}}}+\frac{\lambda_{j}}{l_{\pi_{j}}}+\cdots+\frac{\lambda_{m}}{l_{\pi_{m}}}\right)\right) \\
i, j=1, \ldots, m \text { and } i \neq j, i<j
\end{array}
$$

be the exponential term of (38) and consider the transposition of the terms $i$ and $j$ of the true eigenvalues, i.e.,

$$
\begin{array}{r}
\exp \left(-n\left(\frac{\lambda_{1}}{l_{\pi_{1}}}+\cdots+\frac{\lambda_{i}}{l_{\pi_{j}}}+\frac{\lambda_{j}}{l_{\pi_{i}}}+\cdots+\frac{\lambda_{m}}{l_{\pi_{m}}}\right)\right) \\
i, j=1, \ldots, m \text { and } i \neq j, i<j
\end{array}
$$




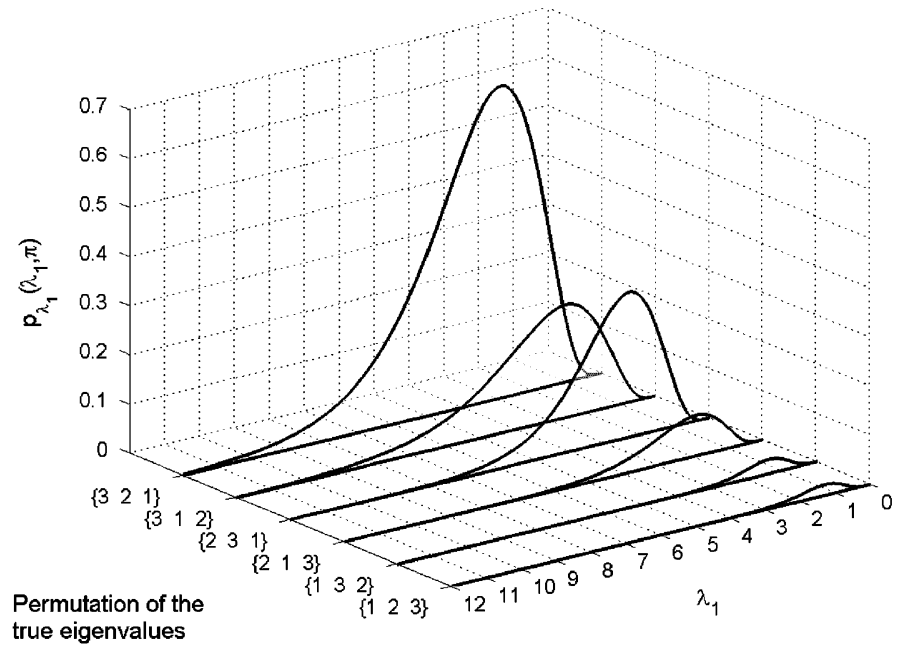

(a)

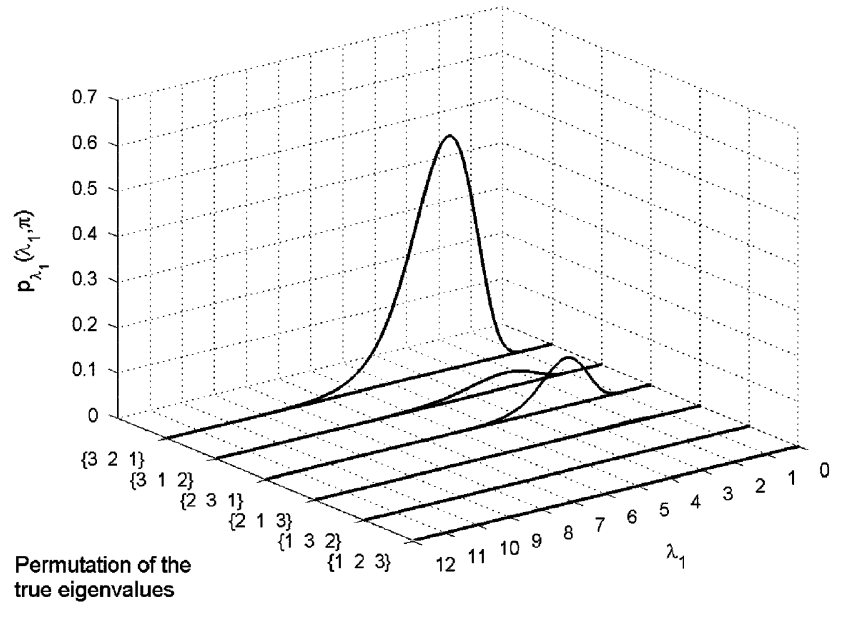

(b)

Fig. 6. Plots of the six sum terms which define $p_{\lambda_{1}}\left(\lambda_{1}\right)$, for 3 and 8 looks, $m=3$ and $\left\{l_{1}, l_{2}, l_{3}\right\}=\{3,2,1\}$. (a) $p_{\lambda_{1}}\left(\lambda_{1}\right) n=3$. (b) $p_{\lambda_{1}}\left(\lambda_{1}\right) n=8$.

where $\lambda_{i} \geq \lambda_{j}$ and $l_{\pi_{i}} \geq l_{\pi_{j}}$. Hence

$$
\begin{aligned}
\frac{\lambda_{i}}{l_{\pi_{i}}}+\frac{\lambda_{j}}{l_{\pi_{j}}} & =\frac{\lambda_{i}}{l_{\pi_{i}} l_{\pi_{j}}}\left(l_{\pi_{j}}+\frac{\lambda_{j}}{\lambda_{i}} l_{\pi_{i}}\right) \\
& =\frac{\lambda_{i}}{l_{\pi_{i}} l_{\pi_{j}}}\left(l_{\pi_{j}}+c l_{\pi_{i}}\right) \\
\frac{\lambda_{i}}{l_{\pi_{j}}}+\frac{\lambda_{j}}{l_{\pi_{i}}} & =\frac{\lambda_{i}}{l_{\pi_{i}} l_{\pi_{j}}}\left(l_{\pi_{i}}+\frac{\lambda_{j}}{\lambda_{i}} l_{\pi_{j}}\right) \\
& =\frac{\lambda_{i}}{l_{\pi_{i}} l_{\pi_{j}}}\left(l_{\pi_{i}}+c l_{\pi_{j}}\right) .
\end{aligned}
$$

Considering $0 \leq c \leq 1$, due to $\infty>\lambda_{1} \geq \lambda_{2} \geq \cdots \geq \lambda_{m} \geq 0$

$$
\begin{gathered}
\frac{\lambda_{i}}{l_{\pi_{i}} l_{\pi_{j}}}\left(l_{\pi_{j}}+c l_{\pi_{i}}\right)-\frac{\lambda_{i}}{l_{\pi_{i}} l_{\pi_{j}}}\left(l_{\pi_{i}}+c l_{\pi_{j}}\right) \\
\quad=\frac{\lambda_{i}}{l_{\pi_{i}} l_{\pi_{j}}}\left(l_{\pi_{j}}(1-c)-l_{\pi_{i}}(1-c)\right) \\
\quad=\frac{\lambda_{i}}{l_{\pi_{i}} l_{\pi_{j}}}(1-c)\left(l_{\pi_{j}}-l_{\pi_{i}}\right) \leq 0 .
\end{gathered}
$$

Finally, by considering (45) in (41) and (42)

$$
\begin{aligned}
& \exp \left(-n\left(\frac{\lambda_{1}}{l_{\pi_{1}}}+\cdots+\frac{\lambda_{i}}{l_{\pi_{i}}}+\frac{\lambda_{j}}{l_{\pi_{j}}}+\cdots+\frac{\lambda_{m}}{l_{\pi_{m}}}\right)\right) \\
& \geq \exp \left(-n\left(\frac{\lambda_{1}}{l_{\pi_{1}}}+\cdots+\frac{\lambda_{i}}{l_{\pi_{j}}}+\frac{\lambda_{j}}{l_{\pi_{i}}}+\cdots+\frac{\lambda_{m}}{l_{\pi_{m}}}\right)\right) .
\end{aligned}
$$

Since a permutation of $\left\{l_{1}, l_{2}, \ldots, l_{m}\right\}$ can be considered as the product of transpositions of the elements $l_{\pi_{i}}$ and $l_{\pi_{j}}$ such that $\lambda_{i} \geq \lambda_{j}$ and $l_{\pi_{i}} \geq l_{\pi_{j}}$, the higher the number of transpositions, the lower the exponential term (in absolute value), with respect to the exponential term of the permutation $\left\{l_{1}, l_{2}, \ldots, l_{m}\right\}$. If one considers now this result within (39), the term of the sum corresponding to the permutation $\left\{l_{1}, l_{2}, \ldots, l_{m}\right\}$, i.e., the term corresponding to the main diagonal in the determinant expression given by (36), is the most important sum term. As a result, the higher the number of transpositions of the permutation $\left\{l_{1}, l_{2}, \ldots, l_{m}\right\}$, the lower the importance of the sum term into $p_{\Xi}(\boldsymbol{\Xi})$. This effect can be clearly observed in Fig. 6 for the distribution of the maximum sample eigenvalue. Finally, since the number of samples $n$ is an argument of the negative exponential functions (41) and (42), the higher the number of looks, the higher this difference. Again, Fig. 6 shows the difference for the number of samples equal to 3 and 8 .

As a result, as the number of samples $n$ increases, all the sum terms of the distribution (39), but the one corresponding to the permutation $\left\{l_{1}, l_{2}, \ldots, l_{m}\right\}$, shall be negligible as they are centered outside the integration interval $\infty>\lambda_{1} \geq \lambda_{2} \geq$ $\cdots \geq \lambda_{m} \geq 0$. Therefore, for $n$ high enough, (39) can be approximated by

$$
\begin{aligned}
p \boldsymbol{\Xi}(\boldsymbol{\Xi})= & K\left(m, n, l_{1}, \ldots, l_{m}\right) \\
& \times \prod_{i=1}^{m} \lambda_{i}^{n-m} \prod_{i<j}^{m}\left(\lambda_{i}-\lambda_{j}\right) \prod_{i=1}^{m} \exp \left(-n \frac{\lambda_{i}}{l_{i}}\right) .
\end{aligned}
$$

Equation (47) represents the approximation, for $n$ high enough, of the joint pdf for the sample eigenvalues of the Hermitian, semipositive definite matrix $\mathbf{Z}_{n}$. A different problem which has been considered in the literature is to determine the joint pdf of the sample eigenvalues when the covariance matrix is symmetric and semipositive definite, i.e., a real matrix. From [31]

$$
\begin{aligned}
p_{\boldsymbol{\Xi}}^{\mathbb{R}}(\boldsymbol{\Xi}) & =K^{\prime}\left(m, n, l_{1}, \ldots, l_{m}\right) \\
& \times \prod_{i=1}^{m} \lambda_{i}^{(n-m-1) / 2} \prod_{i<j}^{m}\left(\lambda_{i}-\lambda_{j}\right)_{0} F_{0}\left(-\frac{n}{2} \boldsymbol{\Sigma}^{-1}, \boldsymbol{\Xi}\right)
\end{aligned}
$$

where, ${ }_{0} F_{0}(\mathbf{A}, \mathbf{B})$ represents the hypergeometric function of double symmetric matrix argument and $K^{\prime}\left(m, n, l_{1}, \ldots, l_{m}\right)$ is a constant which makes the integration of (48) equal to 1 . The function ${ }_{0} F_{0}(\mathbf{A}, \mathbf{B})$ does not present an analytical formula similar to (33) [31], [52]. Hence, it can be only calculated through the evaluation of an infinite series. Nevertheless, ${ }_{0} F_{0}(\mathbf{A}, \mathbf{B})$ presents an approximation formula for large $n$ [31]. Using this approximation, one can prove that (48) can be approximated by

$$
\begin{aligned}
& p_{\boldsymbol{\Xi}}^{\mathbb{R}}(\boldsymbol{\Xi})=K^{\prime \prime}\left(m, n, l_{1}, \ldots, l_{m}\right) \\
& \quad \times \prod_{i=1}^{m} \lambda_{i}^{(n-m-1) / 2} \prod_{i<j}^{m}\left(\lambda_{i}-\lambda_{j}\right)^{1 / 2} \exp \left(-\frac{n}{2} \frac{\lambda_{i}}{l_{i}}\right) .
\end{aligned}
$$


If (49) is compared with (47), one can observe that both pdfs are formally equal. The sole difference is a factor equal to $1 / 2$ in (49) which accounts, basically, for the fact that the off diagonal elements of the symmetric sample covariance matrix contain one independent parameter in the real case, instead of the two for the complex case. It can be concluded that despite the evident differences, for large $n$, the estimation of the true eigenvalues becomes basically the same in the real and in the complex case. In the case of multidimensional SAR data, interest lies in the complex case, as a consequence of the coherent nature of this technology.

Considering (47), Appendix A presents the derivation of the MLE, which is considered as an asymptotic MLE, denoted in the following by A-MLE. The estimation of the true eigenvalues results from the inversion of the following set of equations:

$$
\lambda_{i}=l_{i}+\frac{l_{i}}{n} \sum_{j \neq i}^{m} \frac{l_{j}}{l_{i}-l_{j}}+O\left(n^{-1}\right), \quad i=1,2, \ldots, m
$$

Equation (50) is also found in the real case [31], but with an approximation $O\left(n^{-2}\right)$. In the complex case, the approximation is of order $O\left(n^{-1}\right)$ as a consequence of the approximation introduced at the beginning of this section, leading to (47). The main drawback of (50) is that it can not be inverted. Nevertheless, it gives an expression of the sample eigenvalues $\lambda_{i}$, for $i=1,2, \ldots, m$, as a function for the true eigenvalues $l_{i}$, for $i=1,2, \ldots, m$; under the limitation that (39) can be simplified by (47), which occurs only for $n$ high enough. From (50), it can be concluded that for a large number of looks, $\lambda_{i}=l_{i}$, for $i=1,2, \ldots, m$, i.e., the sample eigenvalues are asymptotically nonbiased estimators of the true eigenvalues, as proved previously. It is clear that the second addition term of (50) consists of the bias of the sample eigenvalues. Despite (50) is only valid for $n$ high enough, in what it follows, its validity shall be extended to the complete range of looks $n$. The limitation for $n$ small is that this characterization has to be considered only from a qualitative point of view.

In order to employ (50) to characterize PolSAR data in the backscattering case, relative eigenvalues are now considered, i.e., $l_{i}$ (rel.) $=l_{i} / l_{1}$, for $i=1,2,3$. This operation allows plotting the biases of the sample eigenvalues, the entropy $H$ and the anisotropy $A$ in 3-D plots. The next list details, on the basis of (50) and for $n=64$, the behavior of the sample eigenvalues, the entropy and the anisotropy.

- $\lambda_{1}$ : As given by Fig. 7, the largest eigenvalue is overestimated, in such a way that the larger the entropy $H$, the higher the bias. The lowest bias corresponds to $H=0$.

- $\lambda_{2}$ : Fig. 8 demonstrates that the second eigenvalue can be overestimated or underestimated. For PolSAR data presenting low entropy, the second eigenvalue is basically underestimated. Nevertheless, for high entropies, the anisotropy has to be considered. For high entropy, in the case of a low anisotropy, the second eigenvalue is overestimated, whereas it is underestimated for high anisotropies.

- $\lambda_{3}$ : From Fig. 9 it can be clearly deduced that this eigenvalue is always underestimated, in such a way, that, the

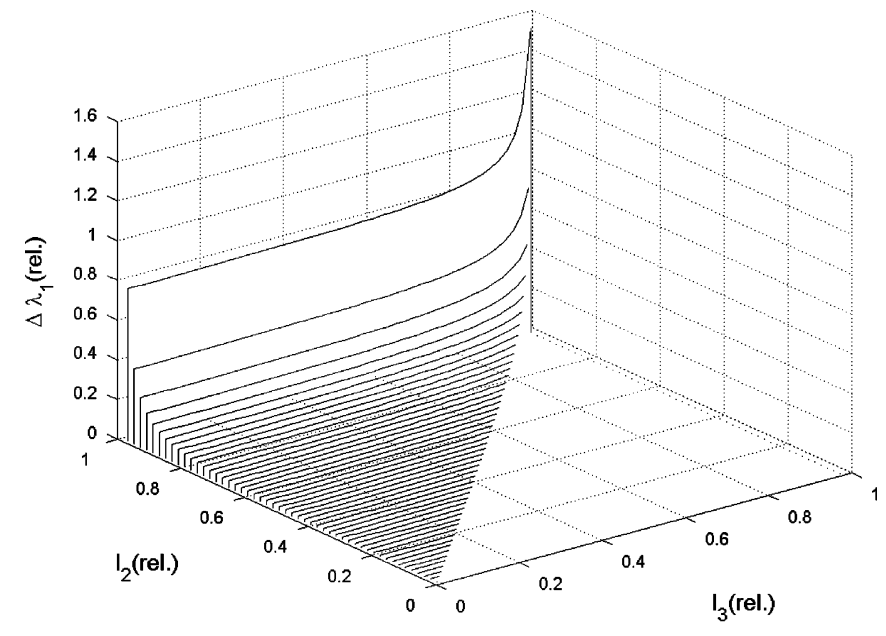

Fig. 7. Bias of the first eigenvalue $\Delta \lambda_{1}(n=64)$.

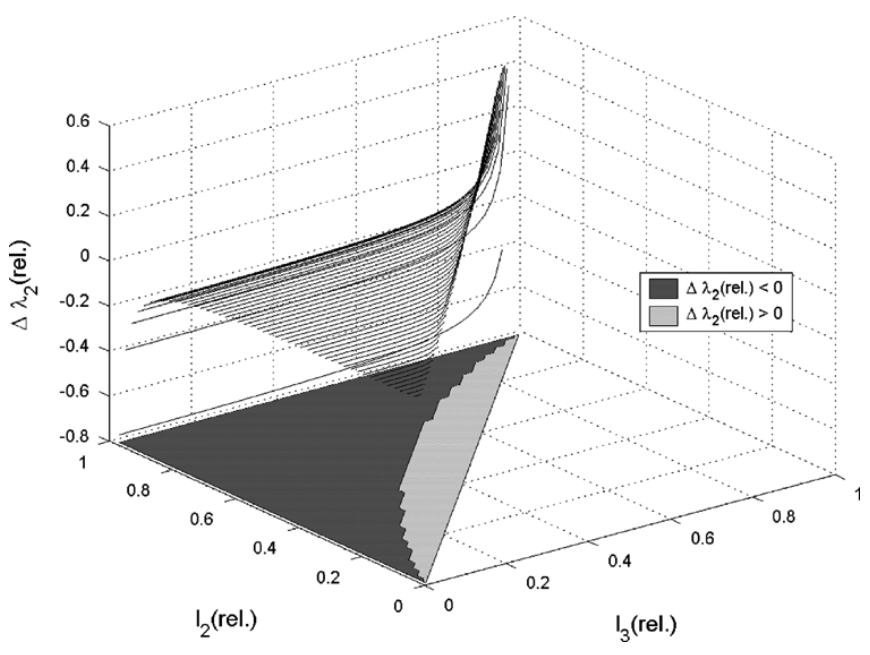

Fig. 8. Bias of the second eigenvalue $\Delta \lambda_{2}(n=64)$.

higher the entropy of the data, the higher the bias (in absolute value).

- $H$ : Fig. 10 depicts the bias of the entropy, from where it can be concluded that the entropy is always underestimated. The largest biases are found for high entropies, or in those cases in which $l_{1}=l_{2}$. The bias presents the smallest value for $H=0$.

- $A$ : The bias of the anisotropy presented in Fig. 11 clearly shows that this quantity can be overestimated or underestimated. From a practical point of view, $A$ can be considered always overestimated. Only in those cases in which $l_{1}=l_{2}$ the anisotropy is underestimated.

2) Eigenvalues Asymptotic Quasi MLE (AQ-MLE): In the process to derive the A-MLE, it is possible to introduce some approximations which can allow finding an approximate solution for the inversion of (50). These approximations are detailed in Appendix B. As a result, an estimator is proposed. This estimator is considered to be asymptotic due to the approximation presented in (47). Additionally, the adjective quasi is introduced in order to reflex the approximations introduced in the process 


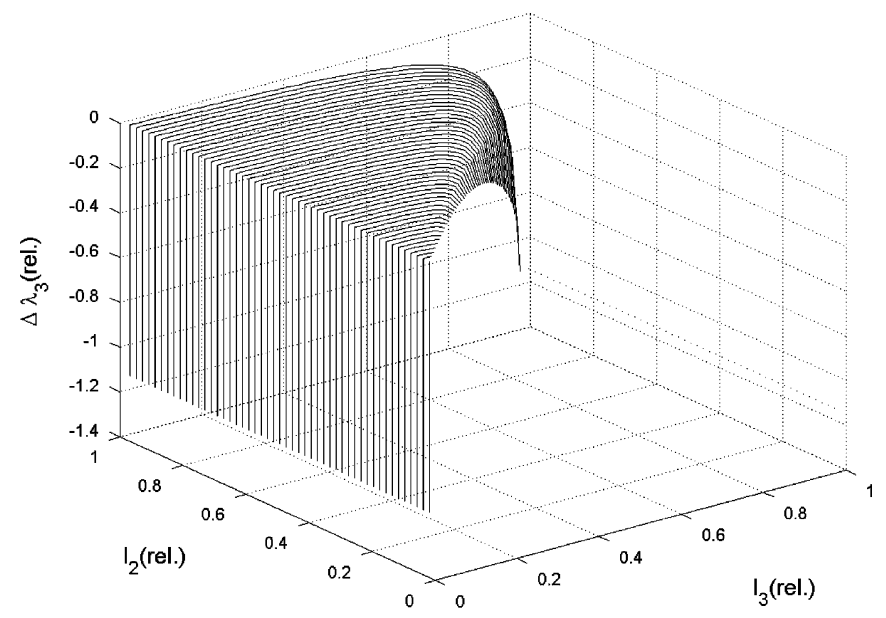

Fig. 9. Bias of the third eigenvalue $\Delta \lambda_{3}(n=64)$.

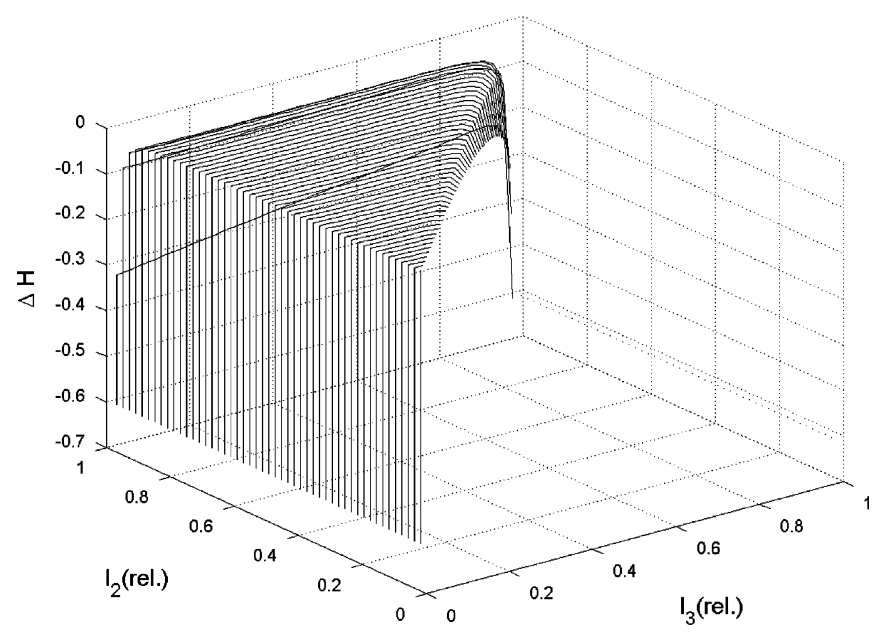

Fig. 10. Bias of the entropy $\Delta H(n=64)$.

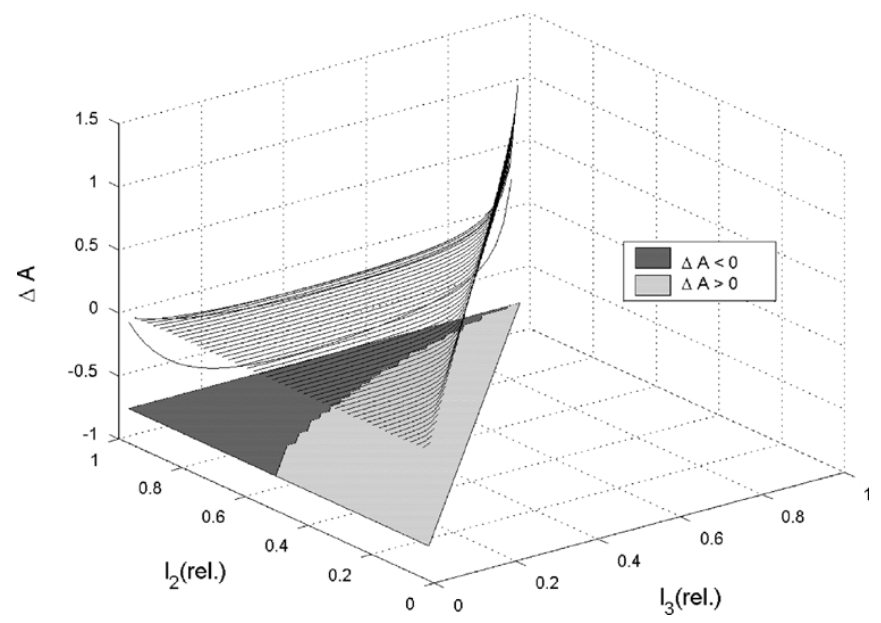

Fig. 11. Bias of the anisotropy $\Delta A(n=64)$.

to derive the MLE. As a result, the AQ-MLE eigenvalues $\hat{l}_{i}$, for $i=1,2, \ldots, m$ are given by

$$
\hat{l}_{i}=\lambda_{i}-\frac{\lambda_{i}}{n} \sum_{j \neq i}^{m} \frac{\lambda_{j}}{\lambda_{i}-\lambda_{j}}-O\left(n^{-1}\right), \quad i=1,2, \ldots, m .
$$

where $\lambda_{i}$, for $i=1,2, \ldots, m$, are the sample eigenvalues derived from $\mathbf{Z}_{n}$. Again, this approximation is of order $O\left(n^{-1}\right)$ due to the approximations considered to arrive to (47). Since (51) is strictly only valid in the asymptotic case, this result has been also found in the real case, although with an approximation order of $O\left(n^{-2}\right)$ [31]. As performed previously, the validity of (51) is extended to any value of $n$. The performance of the AQ-MLE (51) is analyzed by considering the same data studied at the beginning of this section. Fig. 2 presents the correction introduced in the sample eigenvalues (solid curves), whereas Fig. 4 depicts the correction over the entropy $H$ and the anisotropy $A$ values (solid curves). The plots demonstrate that (51) is able to correct the biases when the number of averaged samples is high. However, when the number of looks $n$ is small, the AQ-MLE only corrects part of these biases.

\section{NOTES ON THE $\underline{\alpha}$ PARAMETER}

As indicated in Section II-B, the $H / \underline{\alpha} / A$ decomposition consists of three main parameters. This paper concentrates specifically on $H$ and $A$, which have been derived from the sample eigenvalues. The process followed to obtain the joint pdf of these sample eigenvalues (39) prevents us obtaining any information concerning $\underline{\alpha}$. Nevertheless, for completeness, some insights about the behavior of $\underline{\alpha}$ are now presented. A first option to obtain the statistical behavior of $\underline{\alpha}$ would be to integrate the matrix $\boldsymbol{\Xi}$ in (20) and then, to apply the model presented in (12) to the corresponding eigenvectors. This alternative is characterized by a high mathematical complexity, which suggests itself as a subject of future study. A second choice would be to use Monte Carlo techniques to study the convergence of $\underline{\alpha}$ at the same time as $H / A$.

Nevertheless, the reader is referred to the works presented in [28] and [29]. In these two studies, the authors evaluated, albeit in a qualitative way, the statistical behavior of the $H / \underline{\alpha} / A$ parameters. Both works arrive to the same conclusions. As has been demonstrated quantitatively in the previous sections of this paper, the entropy $H$ is underestimated, whereas the anisotropy is overestimated. In [28], the authors also indicated the dependence of these biases with respect to the level of entropy. The authors of both works indicated that the $\underline{\alpha}$ parameter presents also a bias with respect to its true value. But, in this case, the bias is smaller than in the case of $H$ or $A$. One important conclusion which can be extracted from [28] and [29] is that the eigenvectors, and therefore, the parameters extracted from them, are less affected by the estimation or the speckle noise reduction process.

\section{VALIDATION AND RESULTS}

The sample eigenvalues distribution given by (39) is based on the Gaussian scattering assumption. This hypothesis, despite being only valid to describe homogeneous distributed scatterers, has been widely validated in the literature over real SAR data. Nevertheless, the direct validation of (39) over real data presents the problem that access to the true eigenvalues is not possible due to the uncertainty originated by the eigenvalues biases. As a result, a validation process by means of simulated data is necessary, since access to the true information is granted. 

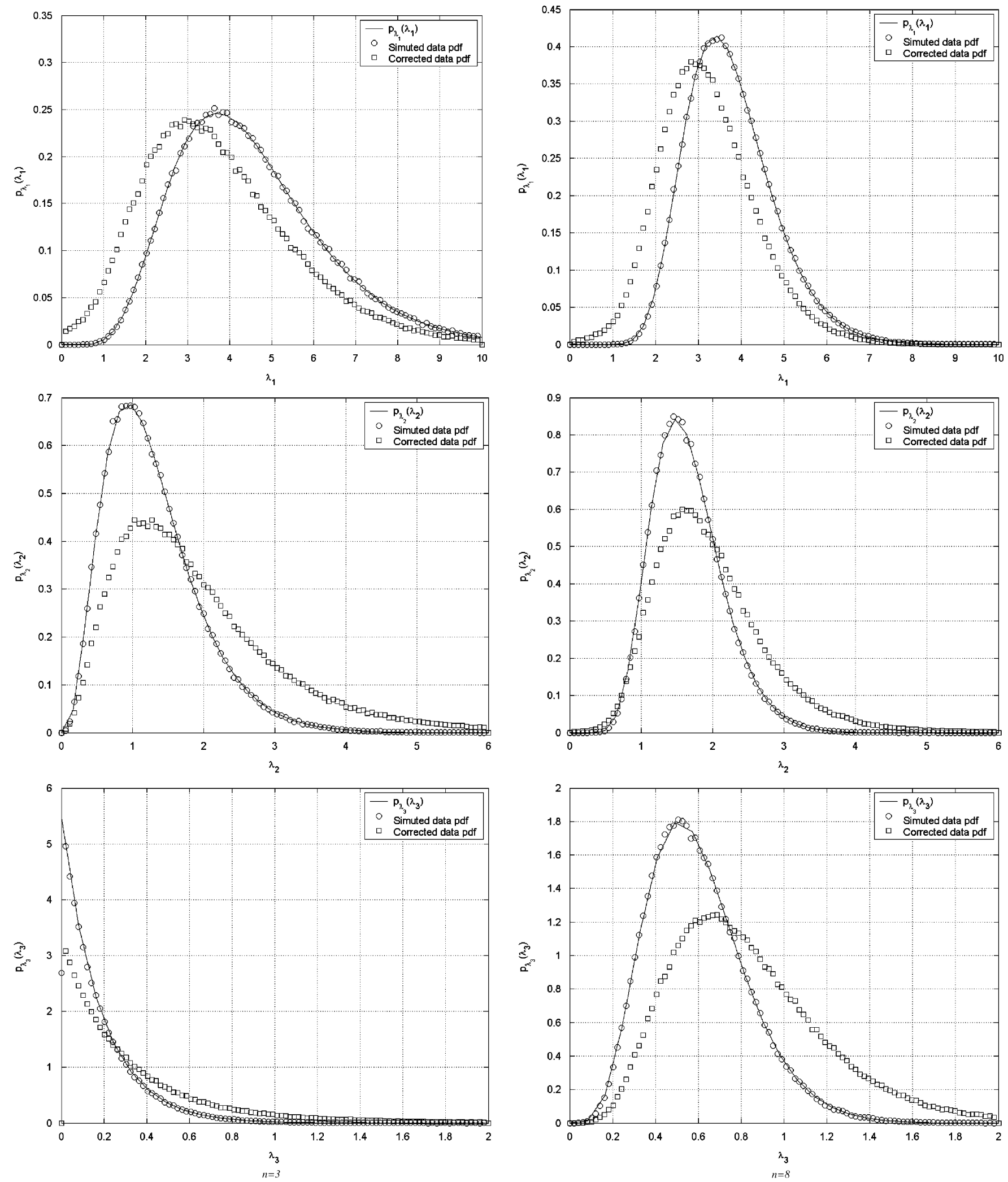

Fig. 12. Probability density distributions comparison $\left\{l_{1}, l_{2}, l_{3}\right\}=\{3,2,1\}$.

\section{A. Simulated Data}

As it has been pointed out in Section V, it is not possible to obtain a simple analytical expression for the distribution of the sample eigenvalues $p_{\lambda_{i}}\left(\lambda_{i}\right)$, for $i=1,2,3$. Hence, the theoretical expressions of these distributions are obtained through a numerical integration of (39). SAR data has been simulated on the basis of the algorithm presented in [60], in which, reflection 
symmetry in a plane normal to the line of sight is considered. Data has been simulated according to the true eigenvalues given in (40), which give rise to the following covariance matrix:

$$
\mathbf{C}=\left[\begin{array}{ccc}
2.5 & 0 & 0.5 \\
0 & 1 & 0 \\
0.5 & 0 & 2.5
\end{array}\right]
$$

in which the copolar correlation is equal to 0.2 . As deduced from (52), $m=3$, whereas two different values have been considered for the number of looks, i.e., $n=3$ and $n=8$. Fig. 12 presents the result of this validation process, where, the continuous line represents the theoretical distributions derived from (39). As it can be observed, the distributions derived from simulated data completely agree with the theoretical values of the distributions.

In the different plots in Fig. 12, the correction given by (51) has been also included in order to visualize its effect over the distribution of the sample eigenvalues. As it can be observed, the correction (51) shifts the distributions of the first eigenvalue to the coordinates center, as the first eigenvalue is overestimated. On the contrary, (51) shifts the distributions of the second and the third eigenvalues away from the coordinates center, as these eigenvalues are underestimated in this case.

\section{B. Experimental Data}

In order to illustrate the results presented in the previous sections of this paper, they are now considered in the frame of experimental SAR data. The data were acquired by the airborne ESAR system, operated by the German Aerospace Center (DLR), in March 2000 in the frame of the Alling-SAR 2000 campaign. Data consist of a L-band, fully polarimetric dataset of a site close to the village of Alling, $25 \mathrm{~km}$ southeast of Munich, Germany. Incidence angle ranges from $25^{\circ}$ in near range to $56^{\circ}$ in far range. The image contains a rural landscape where small-scale agricultural parcels can be easily identified. Some of these fields contain crops in different stages of growth, whereas others are ploughed terrain ready to be planted. Additional forest, small lakes and urban areas are also encountered in the scene.

In what follows, only the behavior of $H$ and $A$ is considered as they allow a compact analysis of the eigenvalues. The dependence on the number of averaged samples or looks to estimate $\mathbf{Z}_{n}$ is studied by considering spatial averaging windows of dimensions ranging from $3 \times 3$ to $11 \times 11$ samples. On the other hand, it has been demonstrated that the eigenvalue biases depend on the eigenvalues themselves (50). Owing to this reason, seven different areas have been selected, which correspond to: shallow water, rough surfaces, vegetated areas, and forested areas, covering all the range of entropy.

Tables I and II present the entropy and anisotropy values derived from the sample eigenvalues $\lambda_{i}$, for $i=1,2, \ldots, m$, in the different areas and for different values of averaged samples. The last column gives the relative difference between the estimated values

$$
\Delta_{\text {rel }}(\%)=100 \frac{\left|H_{9}-H_{121}\right|}{H_{121}} .
$$

For the case of the anisotropy, $H$ is just substituted by $A$ in (53). The first observation which can be deduced from Tables I and II
TABLE I

ENTROPy $(\mathrm{H})$ Estimated From the SAMPLE EigenVAlues $\lambda_{i}$, FOR $i=1,2, \ldots, m$.

\begin{tabular}{l|r|r|r|r|r|r}
\cline { 2 - 7 } & \multicolumn{1}{c}{ 9 looks } & \multicolumn{1}{c|}{ 25 looks } & \multicolumn{1}{c|}{ 49 looks } & \multicolumn{1}{c|}{ 81 looks } & 121 looks & $\Delta_{\text {rel }(\%)}$ \\
\hline Shallow water & 0.1209 & 0.1280 & 0.1297 & 0.1309 & 0.1355 & 10.7 \\
\hline Rough Surf. 1 & 0.2816 & 0.3073 & 0.3149 & 0.3182 & 0.3203 & 12.1 \\
\hline Rough Surf. 2 & 0.3468 & 0.3951 & 0.4110 & 0.4175 & 0.4211 & 17.6 \\
\hline Vegetated Surf. 1 & 0.6423 & 0.7777 & 0.8286 & 0.8525 & 0.8656 & 25.8 \\
\hline Vegetated Surf. 2 & 0.3126 & 0.3506 & 0.3658 & 0.3735 & 0.3792 & 17.7 \\
\hline Forest 1 & 0.6802 & 0.8284 & 0.8872 & 0.9166 & 0.9332 & 27.1 \\
\hline Forest 2 & 0.6892 & 0.8352 & 0.8898 & 0.9170 & 0.9325 & 26.1 \\
\hline
\end{tabular}

TABLE II

ANisotropy (A) ESTIMATED From the SAMPLE EIgENVALUeS $\lambda_{i}$, FOR $i=1,2, \ldots, m$.

\begin{tabular}{l|c|r|r|r|r|r}
\cline { 2 - 7 } & \multicolumn{1}{c|}{ 9 looks } & 25 looks & 49 looks & 81 looks & 121 looks & $\Delta_{\text {rel }} \%$ \\
\hline Water & 0.5167 & 0.3385 & 0.2588 & 0.2159 & 0.1930 & 167.7 \\
\hline Rough Surf. 1 & 0.5487 & 0.3894 & 0.3180 & 0.2794 & 0.2561 & 114.3 \\
\hline Rough Surf. 2 & 0.5609 & 0.3897 & 0.3003 & 0.2475 & 0.2124 & 164.1 \\
\hline Vegetated Surf. 1 & 0.5702 & 0.4481 & 0.3997 & 0.3786 & 0.3682 & 54.9 \\
\hline Vegetated Surf. 2 & 0.5631 & 0.3777 & 0.2885 & 0.2377 & 0.2028 & 164.4 \\
\hline Forest 1 & 0.5266 & 0.3724 & 0.2973 & 0.2838 & 0.2261 & 132.9 \\
\hline Forest 2 & 0.5172 & 0.3555 & 0.2777 & 0.2320 & 0.2020 & 156 \\
\hline
\end{tabular}

is that the larger the number of averaged samples, the higher the entropy and the lower the anisotropy. This behavior of the real measurements reflects the theoretical behavior demonstrated in Section V, where it was shown that the entropy derived from the sample eigenvalues is underestimated with respect to its real value, whereas anisotropy is basically overestimated. Consequently, these results confirm the fact that the sample eigenvalues consist of biased estimators of the true eigenvalues. Nevertheless, a more in-depth analysis of Tables I and II confirms also the asymptotic nature of the sample eigenvalues, the entropy and the anisotropy.

With respect to the entropy values presented in Table I, from low entropy areas, i.e., shallow water and rough surface areas, it can be observed that they present the lowest variation with respect to the number of looks. This validates the theoretical result presented in Fig. 10, which showed that the entropy bias is proportional to the entropy itself, in such a way that higher entropies present the higher biases. The special case of rough surfaces was specifically analyzed by the authors in [28], arriving to the same conclusions. In the same way, Table II corroborates the anisotropy bias behavior given at Fig. 11. Nevertheless, despite the overestimation of the anisotropy can be clearly observed, a direct relation with the entropy values can not be established. Finally, if Table I is compared with Table II, one can clearly deduce that the anisotropy presents a larger variation with respect to the number of looks. This fact suggests that a larger number of looks is necessary in order to estimate a reliable anisotropy value, compared with the entropy.

The sample eigenvalues derived in the areas presented in Tables I and II have been also employed to derive the AQ-MLE of the true eigenvalues; see (51). Results are presented in Tables III and IV, respectively. First of all, if one compares the values of $H$ and $A$ derived from the sample eigenvalues at Tables I and II with those derived with the AQ-MLE approach, Tables III and IV, it is clear that this approach is only able to correct part of the eigenvalues biases, as it has been derived under the asymptotic hypothesis. A closer look to the entropy 
TABLE III

ENTROPY (H) ESTIMATED FROM THE AQ-MLE EIGENVALUES $\hat{l}_{i}$, FOR $i=1,2, \ldots, m$

\begin{tabular}{l|c|r|r|r|r|r}
\cline { 2 - 7 } & \multicolumn{1}{c|}{9 looks } & \multicolumn{1}{c|}{25 looks } & \multicolumn{1}{c|}{ 49 looks } & \multicolumn{1}{c|}{ 81 looks } & 121 looks & \multicolumn{1}{c}{$\Delta_{\text {rel }}(\%)$} \\
\hline Water & 0.1325 & 0.1324 & 0.1320 & 0.1323 & 0.1345 & 1.5 \\
\hline Rough Surf. 1 & 0.3077 & 0.3179 & 0.3205 & 0.3217 & 0.3226 & 4.7 \\
\hline Rough Surf. 2 & 0.3787 & 0.4088 & 0.4183 & 0.4220 & 0.4242 & 10.7 \\
\hline Vegetated Surf. 1 & 0.6951 & 0.8025 & 0.8427 & 0.8615 & 0.8719 & 20.3 \\
\hline Vegetated Surf. 2 & 0.3415 & 0.3627 & 0.3723 & 0.3776 & 0.3820 & 10.6 \\
\hline Forest 1 & 0.7350 & 0.8541 & 0.9018 & 0.9257 & 0.9397 & 21.7 \\
\hline Forest 2 & 0.7446 & 0.8600 & 0.9044 & 0.9264 & 0.9390 & 20.7 \\
\hline
\end{tabular}

TABLE IV

ANisotropy (H) Estimated From the AQ-MLE EIgENVALUES $\hat{l}_{i}$, FOR $i=1,2, \ldots, m$

\begin{tabular}{l|r|r|r|r|r|r}
\cline { 2 - 7 } & \multicolumn{1}{c|}{ 9 looks } & \multicolumn{1}{c|}{ 25 looks } & 49 looks & 81 looks & 121 looks & \multicolumn{1}{c}{$\Delta_{\text {rel }}(\%)$} \\
\hline Water & 0.4294 & 0.2751 & 0.2135 & 0.1825 & 0.1675 & 156.3 \\
\hline Rough Surf. 1 & 0.4710 & 0.3365 & 0.2819 & 0.2533 & 0.2364 & 99.3 \\
\hline Rough Surf. 2 & 0.4881 & 0.3379 & 0.2624 & 0.2184 & 0.1894 & 157.8 \\
\hline Vegetated Surf. 1 & 0.5122 & 0.4169 & 0.3813 & 0.3671 & 0.3606 & 42.1 \\
\hline Vegetated Surf. 2 & 0.4892 & 0.3225 & 0.2483 & 0.2078 & 0.1781 & 174.7 \\
\hline Forest 1 & 0.4292 & 0.3303 & 0.2688 & 0.2329 & 0.2097 & 104.7 \\
\hline Forest 2 & 0.4483 & 0.3105 & 0.2455 & 0.2076 & 0.1826 & 145.5 \\
\hline
\end{tabular}

values demonstrates that the AQ-MLE approach makes possible to reduce the number of looks in order to estimate reliable entropy values. In the case that the covariance matrix $\mathbf{Z}_{n}$ is obtained by $11 \times 11$ pixel averaging windows, the results at Tables I and III are quite the same, i.e., the AQ-MLE does not correct the entropy value. Nevertheless, if one compares the same values in the tables referring to the anisotropy, i.e., Tables II and IV, it can be clearly observed that the AQ-MLE approach introduces a correction in the anisotropies. This fact reinforces the idea suggesting that the anisotropy needs from more samples than the entropy, in order to estimate a reliable value. This can be explained by the fact that the anisotropy value is obtained from the second and the third eigenvalues of $\mathbf{Z}_{n}$, which are more affected by the speckle and thermal noises.

Finally, we note that the estimation problems are worse for forested and heavily vegetated terrain (high entropy) than for nonvegetated surfaces (low entropy). Hence it can be concluded that quantitative parameter estimation using PolSAR alone is probably better suited to surface rather than vegetation problems. It is important to point out that this high entropy for vegetation is due to the random scattering environment itself and not to a system effect such as noise. Hence in order to secure more robust estimates, the "entropy" of vegetation scattering must somehow be reduced. One way to do this is to employ polarimetric interferometry (PolInSAR) where the coherence over vegetated regions can be controlled by baseline selection and parameter sensitivity still provided by polarization diversity. Future studies will consider bias and estimation issues in this higher dimensional environment.

\section{CONCLUSION}

In this paper, we have presented an in-depth statistical analysis of the eigendecomposition of the complex Hermitian covariance and coherency matrices, as well as for the associated
$H / \underline{\alpha} / A$ decomposition. From the detailed analysis of the existing literature, it was observed that this problem presents several similarities with the study of real symmetric matrices. In this study, we have extended existing results in order to obtain a simple expression for the distribution of the sample eigenvalues. From the study of this expression, it has been possible to conclude that the sample eigenvalues, the entropy and the anisotropy are asymptotically nonbiased estimators of the corresponding true values. Finally, these biases can not be completely corrected as they also depend on the value of the signals to recover.

The existence of the biases for the sample eigenvalues is critical when quantitative remote sensing is addressed, since they have to be seen as a source of error, which shall be transmitted to the physical parameters obtained from them. In order to correct the effects of these biases, an asymptotic quasi maximum-likelihood estimator is proposed. The application of this estimator to real data demonstrates, as shown theoretically, its incapability to fully correct all the biases. Nevertheless, the biases are no so critical for classification applications, since in these cases, the effects of the biases can be mitigated by the fact that other parameters, such as the span, are also considered. Finally, we have considered the effect of the number of averaged samples in order to estimate the entropy $H$ and the anisotropy $A$. It is demonstrated that the minimum number of looks to consider these quantities unbiased differ in such a way since the anisotropy are more affected by the sample eigenvalues biases than the entropy.

The main conclusion to be extracted from the study presented in this paper is that PolSAR speckle noise presents a big impact on quantitative physical parameters estimation, especially in high entropy environments. We have considered in the previous sections the eigen- and the $H / \underline{\alpha} / A$ decompositions as tools to retrieve the physical parameters. Since there exist alternative approaches to derive the physical information of interest, one can speculate about the possibility that these alternative techniques shall be also affected by the same type of effects shown in this paper. The bottom line of all this work is that the problem of speckle noise in multidimensional SAR systems needs further research work in order to determine completely its effects on useful data and with the objective to improve its filtering and removal.

\section{APPENDIX A}

This Appendix contains the derivation of the MLE of the eigenvalues, given its joint asymptotic distribution (47). The log-likelihood function of (47) is

$$
\begin{gathered}
\ln \left(p_{\boldsymbol{\Xi}}(\boldsymbol{\Xi}, \boldsymbol{\Sigma})\right)=\ln \left(\prod_{i=1}^{m} \lambda_{i}^{n-m}\right)+\ln \left(\prod_{i<j}^{m}\left(\lambda_{i}-\lambda_{j}\right)\right) \\
+\ln \left(K\left(m, n, l_{1}, \ldots, l_{m}\right) \prod_{i=1}^{m} \exp \left(-n \frac{\lambda_{i}}{l_{i}}\right)\right) .
\end{gathered}
$$


Considering the value of the constant $K$, as given by (37), the maximization process of (A.1) is given in (A.2), shown at the bottom of the page. The derivative of the term $A$ is

$$
\begin{aligned}
\frac{\partial}{\partial l_{q}} \sum_{i<j}^{m} \ln \left(\frac{l_{i} l_{j}}{l_{i}-l_{j}}\right) & =\sum_{\substack{j=1 \\
j \neq q}}^{m} \frac{l_{j}}{l_{q}\left(l_{j}-l_{q}\right)} \\
& =-\sum_{\substack{j=1 \\
j \neq q}}^{m} \frac{l_{j}}{l_{q}\left(l_{q}-l_{j}\right)}, \quad q=1,2, \ldots, m
\end{aligned}
$$

In the same way, the derivative of the term $B$ is

$$
\begin{aligned}
\frac{\partial}{\partial l_{q}} \sum_{i=1}^{m} \ln \left(l_{i}^{-n} \exp \left(-n \frac{\lambda_{i}}{l_{i}}\right)\right)=-\frac{n}{l_{q}} & +\frac{n \lambda_{i}}{l_{q}^{2}}, \\
q & =1,2, \ldots, m .
\end{aligned}
$$

In order to find the maximum of (A.1), the addition of (A.3) and (A.4) is considered

$$
-\frac{n}{l_{i}}+\frac{n \lambda_{i}}{l_{i}^{2}}-\sum_{j \neq i}^{m} \frac{l_{j}}{l_{i}\left(l_{i}-l_{j}\right)}=0, \quad i=1,2, \ldots, m .
$$

Consequently, one derives the following system of equations:

$$
\lambda_{i}=l_{i}+\frac{l_{i}}{n} \sum_{j \neq i}^{m} \frac{l_{j}}{l_{i}-l_{j}}, \quad i=1,2, \ldots, m .
$$

\section{APPENDIX B}

This Appendix contains the approximations in the process to derive the A-MLE, detailed in Appendix A, which allow finding (51). Due to the presence of a bias in the sample eigenvalues, one can consider that the true eigenvalues $l_{i}$ for $i=1, \ldots, m$ and its estimators $\lambda_{i}$ are for $i=1, \ldots, m$ will present a difference. Therefore, in the term labeled as $A$ in (A.2) it can be written

$\prod_{i<j}^{m} \frac{l_{i} l_{j}}{l_{i}-l_{j}}=\prod_{i<j}^{m}\left(\frac{1}{l_{j}}-\frac{1}{l_{i}}\right)^{-1}$

$$
\frac{1}{l_{j}}-\frac{1}{l_{i}}=\left(\frac{1}{\lambda_{j}}-\frac{1}{\lambda_{i}}\right)\left(1+\frac{\left(\frac{1}{l_{j}}-\frac{1}{\lambda_{j}}\right)-\left(\frac{1}{l_{i}}-\frac{1}{\lambda_{i}}\right)}{\frac{1}{\lambda_{j}}-\frac{1}{\lambda_{i}}}\right) .
$$

Consequently, the derivative of the term $A$ in (A.2) takes the expression given in (B.3), shown at the bottom of the page. If one considered the expansion of the logarithm function into its polynomial series expansion, i.e., its Taylor series

$$
\ln (1+x)=\sum_{n=1}^{\infty}(-1)^{n+1} \frac{x^{n}}{n}
$$

the derivative in (B.3) can be approximated by the next expression

$$
\begin{aligned}
\frac{\partial}{\partial l_{q}} \sum_{i<j}^{m} \ln \left(\frac{l_{i} l_{j}}{l_{i}-l_{j}}\right) & \simeq-\sum_{\substack{j=1 \\
j \neq q}}^{m} \frac{1}{l_{q}^{2}} \frac{\lambda_{q} \lambda_{j}}{\lambda_{q}-\lambda_{j}} \\
& =-\frac{\lambda_{q}}{l_{q}^{2}} \sum_{\substack{j=1 \\
j \neq q}}^{m} \frac{\lambda_{q}}{\lambda_{q}-\lambda_{j}}, \quad q=1,2, \ldots, m .
\end{aligned}
$$

If the simplification presented by (B.5) of the term $A$ of (A.2) is introduced into this equation, one can easily arrive to the next expression

$$
\begin{gathered}
\frac{\partial \ln \left(p_{\Xi}(\boldsymbol{\Xi}, \boldsymbol{\Sigma})\right)}{\partial l_{q}}=-\frac{n}{l_{q}}+\frac{n \lambda_{q}}{l_{q}^{2}}-\frac{\lambda_{q}}{l_{q}^{2}} \sum_{\substack{j=1 \\
j \neq q \\
j \neq q}}^{m} \frac{\lambda_{j}}{\lambda_{q}-\lambda_{j}} \\
q=1,2, \ldots, m .
\end{gathered}
$$

Finally, if (B.6) is make zero, it is derived that

$$
l_{i}=\lambda_{i}-\frac{\lambda_{i}}{n} \sum_{j \neq i}^{m} \frac{\lambda_{j}}{\lambda_{i}-\lambda_{j}}, \quad i=1,2, \ldots, m .
$$

Therefore, the estimators of the true eigenvalues $l_{i}$ for $i=$ $1,2, \ldots, m$, i.e., $\hat{l}_{i}$ for $i=1,2, \ldots, m$, considering the sample

$$
\begin{aligned}
\left.\frac{\partial \ln \left(p_{\boldsymbol{\Xi}}(\boldsymbol{\Xi}, \boldsymbol{\Sigma})\right)}{\partial l_{q}}\right|_{q=1, \ldots, m} & =\left.\frac{\partial}{\partial l_{q}} \ln \left(\prod_{i<j}^{m} \frac{l_{i} l_{j}}{l_{i}-l_{j}}\right)\right|_{q=1, \ldots, m}+\left.\frac{\partial}{\partial l_{q}}\left(\ln \prod_{i=1}^{m} l_{i}^{-n} \exp \left(-n \frac{\lambda_{i}}{l_{i}}\right)\right)\right|_{q=1, \ldots, m} \\
& =\underbrace{\left.\frac{\partial}{\partial l_{q}} \sum_{i<j}^{m} \ln \left(\frac{l_{i} l_{j}}{l_{i}-l_{j}}\right)\right|_{q=1, \ldots, m}}_{A}+\underbrace{\left.\frac{\partial}{\partial l_{q}} \sum_{i=1}^{m} \ln \left(l_{i}^{-n} \exp \left(-n \frac{\lambda_{i}}{l_{i}}\right)\right)\right|_{q=1, \ldots, m}}_{B}
\end{aligned}
$$

$$
\begin{aligned}
\left.\frac{\partial}{\partial l_{q}} \sum_{i<j}^{m} \ln \left(\frac{l_{i} l_{j}}{l_{i}-l_{j}}\right)\right|_{q=1, \ldots, m} & =\left.\frac{\partial}{\partial l_{q}} \sum_{i<j}^{m} \ln \left(\frac{1}{l_{j}}-\frac{1}{l_{i}}\right)^{-1}\right|_{q=1, \ldots, m} \\
& =-\left.\frac{\partial}{\partial l_{q}} \sum_{i<j}^{m} \ln \left(\left(\frac{1}{\lambda_{j}}-\frac{1}{\lambda_{i}}\right) \times\left(1+\frac{\left(\frac{1}{l_{j}}-\frac{1}{\lambda_{j}}\right)-\left(\frac{1}{l_{i}}-\frac{1}{\lambda_{i}}\right)}{\frac{1}{\lambda_{j}}-\frac{1}{\lambda_{i}}}\right)\right)\right|_{q=1, \ldots, m}
\end{aligned}
$$


eigenvalues $\lambda_{i}$ for $i=1,2, \ldots, m$ as the available data, take the expression

$$
\hat{l}_{i}=\lambda_{i}-\frac{\lambda_{i}}{n} \sum_{j \neq i}^{m} \frac{\lambda_{j}}{\lambda_{i}-\lambda_{j}}, \quad i=1,2, \ldots, m .
$$

\section{ACKNOWLEDGMENT}

The authors would like to thank the reviewers for their useful comments and suggestions which help to improve the paper.

\section{REFERENCES}

[1] J. C. Curlander and R. N. McDonough, Synthetic Aperture Radar: Systems and Signal Processing. New York: Wiley, 1991.

[2] J. W. Goodman, "Some fundamental properties of speckle," J. Opt. Soc. Amer., vol. 66, no. 11, pp. 1145-1149, Nov. 1976

[3] J. S. Lee, "Speckle analysis and smoothing of synthetic aperture radar images," Comput. Graph. Image Process., vol. 17, pp. 24-32, 1981.

[4] A. Lopes, R. Touzi, and E. Nezry, "Adaptive speckle filters and scene heterogeneity," IEEE Trans. Geosci. Remote Sens., vol. 25, no. 6, pp. 992-1000, Nov. 1990.

[5] R. J. Q. Tough, D. Blacknell, and S. Quegan, "A statistical description of polarimetric and interferometric synthetic aperture radar data," in Proc. R. Soc. London A, vol. 449, 1995, pp. 567-589.

[6] J. S. Lee, K. W. Hoppel, and S. A. Mango, "Intensity and phase statistics of multilook polarimetric interferometric SAR imagery," IEEE Trans. Geosci. Remote Sens., vol. 32, no. 5, pp. 1017-1027, Sep. 1994.

[7] C. Oliver and S. Quegan, Understanding Synthetic Aperture Radar Images. Boston, MA: Artech House, 1998.

[8] R. Touzi and A. Lopes, "Statistics of the Stokes parameters and of the complex coherence parameters in one-look and multilook speckle fields," IEEE Trans. Geosci. Remote Sens., vol. 34, no. 2, pp. 519-531, Mar. 1996.

[9] C. López-Martínez and X. Fàbregas, "Polarimetric SAR speckle noise model," IEEE Trans. Geosci. Remote Sens., vol. 41, no. 10, pp. 2232-2242, Oct. 2003.

[10] F. T. Ulaby and C. Elachi, Radar Polarimetry for Geoscience Applications. Norwood, MA: Artech House, 1990.

[11] S. R. Cloude, "A new method for characterizing depolarization effects in radar and optical remote sensing," in Proc. IGARSS, vol. 2, Sydney, Australia, 2001, pp. 910-912.

[12] J. R. Huynen, "Phenomenological theory of radar targets," Ph.D. dissertation, Tech. Univ. Delft, Delft, The Netherlands, 1970.

[13] S. R. Cloude and E. Pottier, "A review of target decomposition theorems in radar polarimetry," IEEE Trans. Geosci. Remote Sens., vol. 34, no. 2, pp. 498-518, Mar. 1996.

[14] S. R. Cloude, "Group theory and polarization algebra," OPTIK, vol. 75, no. 1 , pp. 26-36, 1986.

[15] A. Freeman and S. L. Durden, "A three component scattering model for polarimetric SAR data," IEEE Trans. Geosci. Remote Sens., vol. 33, no. 3, pp. 963-973, May 1998.

[16] E. Krogager, "A new decomposition of the radar target scattering matrix," IEE Electron. Lett., vol. 26, no. 18, pp. 1525-1526, 1990.

[17] W. L. Cameron and L. K. Leung, "Feature motivated polarization scattering matrix decomposition," in Proc. IEEE Int. Radar Conf., Arlington, VA, May 7-10, 1990, pp. 549-557.

[18] S. R. Cloude, "Radar target decomposition theorems," Electron. Lett., vol. 21, no. 1, pp. 22-24, Jan. 1985.

[19] S. R. Cloude and E. Pottier, "An entropy based classification scheme for land applications of polarimetric SAR," IEEE Trans. Geosci. Remote Sens., vol. 35, no. 1, pp. 68-78, Jan. 1997.

[20] E. Pottier and J. S. Lee, "Unsupervised classification of PolSAR images based on the complex wishart distribution and the $\mathrm{H} / \mathrm{A} / \underline{\alpha}$ polarimetric decomposition theorem," in Proc. EUSAR, Munich, Germany, May 2000.

[21] K. R. Czuchlewski, J. K. Weissel, and Y. Kim, "Polarimetric synthetic aperture radar study of the Tsaoling landslide generated by the 1999 Chi-Chi earthquake, Taiwan," J. Geophys. Res., vol. 108, no. F1, pp. 7.1-7.11, 2003.

[22] J. K. Weissel, K. R. Czuchlewski, and Y. Kim, "Synthetic aperture radar (SAR)-based mapping of volcanic flows: Manam Island, Papua New Guinea," Natural Hazards Earth Syst. Sci., vol. 4, pp. 339-346, 2004.
[23] S. R. Cloude, "Eigenvalue parameters for surface roughness studies," in Proc. SPIE Conf. Polarization: Measurement, Analysis, and Remote Sensing II, vol. 3754, Denver, CO, Jul. 1999.

[24] I. Hajnsek, E. Pottier, and S. R. Cloude, "Inversion of surface parameters fom polarimetric SAR," IEEE Trans. Geosci. Remote Sens., vol. 41, no. 4, pp. 727-744, Apr. 2003.

[25] S. R. Cloude, I. Hajnsek, and K. P. Papathanassiou, "An eigenvector method for the extraction of surface parameters in polarimetric SAR," in Proc. CEOS, Toulouse, France, Oct. 1999.

[26] S. Allain, L. Ferro-Famil, and E. Pottier, "Surface parameters retrieval from polarimetric and multi-frequency SAR data," in Proc. IGARSS, vol. 2, Toulouse, France, 2003, pp. 1417-1419.

[27] J. M. Lopez-Sanchez, "Analysis and estimation of biophysical parameters of vegetation by radar polarimetry," Ph.D. dissertation, Tech. Univ. of Valencia, Valencia, Spain, 1999.

[28] C. Lopez-Martinez, E. Pottier, I. Hajnsek, J.-S. Lee, and X. Fabregas, "Polarimetric speckle noise effects in quantitative physical parameters retrieval," in Proc. EUSAR, Ulm, Germany, May 2004.

[29] J. S. Lee, D. L. Schuler, and T. L. Ainsworth, "Scattering model based speckle filtering of polarimetric SAR data," in Proc. EUSAR, Ulm, Germany, May 2004.

[30] I. Sikaneta, C. Gierull, and J. Y. Chouinard, "Metrics for SAR-GMTI based on the eigen decomposition of the sample covariance matrix," in Proc. RADAR 2003, Adelaide, Australia, Sep. 2003.

[31] R. J. Muirhead, Aspects of Multivariate Statistical Theory. New York: Wiley, 1982.

[32] D. H. O. Bebbington, "Target vectors-spinorial concepts," in Proc. 2nd Int. Workshop on Radar Polarimetry, Nantes, France, Sept. 1992, pp. $26-36$.

[33] S. R. Cloude and K. P. Papathanassiou, "Polarimetric SAR interferometry," IEEE Trans. Geosci. Remote Sens., vol. 36, no. 5, pp. 1551-1565, Sep. 1998.

[34] R. M. A. Azzam and N. M. Bashara, Ellipsometry and Polarized Light The Netherlands: Elsevier, 1987.

[35] J. A. Kong, "Polarimetric remote sensing," in Progr. Electromagn. Res.. New York: Elsevier, 1990, vol. 3.

[36] G. De Grandi, J. S. Lee, D. Schuler, and E. Nezry, "Texture and speckle statistics in polarimetric SAR synthesized images," IEEE Trans. Geosci. Remote Sens., vol. 41, no. 9, pp. 2070-2088, Sep. 2003.

[37] C. Oliver and S. Quegan, Understanding Synthetic Aperture Radar Images. Boston, MA: Artech House, 1998.

[38] N. R. Goodman, "Statistical analysis based on a certain multivariate complex gaussian distribution (an introduction)," Ann. Math. Statist. vol. 34, pp. 152-177, 1963.

[39] E. Cartan, Leçons sur Les Invariants Integraux. Paris, France: Hermann, 1922.

[40] — - Formes Differentielles. Paris, France: Hermann, 1967.

[41] H. Flanders, Differential Forms with Applications to the Physical Sciences, Mathematics in Science and Engineering. New York: Academic, 1963, vol. 11.

[42] H. Grassmann, Extension Theory, History of Mathematics. Providence, RI: Amer. Math. Soc., 2000, vol. 19. First Published in 1861.

[43] C. E. Porter, Statistical Theories of Spectra: Fluctuations. New York: Academic, 1965

[44] A. Haar, "Der massbegiff in der theorie der kotinuierlichen gruppen," Ann. Math., vol. 34, pp. 374-385, 1933.

[45] V. Girko, Theory of Random Determinants. Amsterdam, The Netherlands: Kluwer, 1990

[46] S. Helgason, Differential Geometry and Symmetric Spaces. New York: Academic, 1962

[47] W. Miller, Lie Theory and Special Functions. New York: Academic, 1968.

[48] A. T. James, "Zonal polynomials of the real positive definite symmetric matrices," Ann. Math., vol. 74, no. 3, pp. 456-469, Nov. 1961.

[49] A. Takemura, Zonal Polynomials: Inst. Math. Stat., 1984, vol. 4, Lecture Notes-Monograph Series.

[50] I. G. MacDonald, Symmetric Functions and Hall Polynomials, 2nd ed. Oxford, UK: Claredon, 1995.

[51] A. T. James, "Distributions of matrix variates and latent roots derived from normal samples," Ann. Math. Stat., vol. 35, pp. 475-501, 1964.

[52] K. I. Gross and D. St. P. Richards, "Special functions of matrix argument I: Algebraic induction, zonal polynomials, and hypergeometric functions," Trans. Amer. Math. Soc., vol. 301, no. 2, pp. 781-811, Jun. 1987.

[53] R. Gutierrez, J. Rodriguez, and J. Saez, "Approximation of hypergeometric functions with matricial argument through their development in series of zonal polynomials," Electron. Trans. Numer. Anal., vol. 11, pp. 121-130, 2000. 
[54] G. L. Lamb, Elements of Soliton Theory. New York: Wiley, 1980.

[55] K. Takasaki, "Initial value problem for the toda lattice hierarchy," $A d v$. Stud. Pure Math., vol. 4, pp. 139-163, 1984.

[56] T. Takebe, "Representation theoretical meaning of initial value problem for the toda latice hierarchy I," Lett. Math. Phys., vol. 21, pp. 77-84, 1991.

[57] A. Y. Orlov and D. M. Scherbi, "Hypergeometric solutions of soliton equations," Theoret. Math. Phys., vol. 128, no. 1, pp. 906-926, 2001.

[58] S. C. Milne, "Summation theorems for basic hypergeometric series of Schur function argument," in Progress in Approximation Theory. New York: Springer-Verlag, 1992, pp. 51-77.

[59] A. Yu. Orlov and D. M. Scherbin, "Multivariate hypergeometric functions as tau-functions of toda lattice and Kadomtsev-Petviashvili equation," Phys. D: Nonlinear Phenomena, vol. 152-153, pp. 51-65, May 2001.

[60] L. M. Novak and M. C. Burl, "Optimal speckle reduction polarimetric SAR imagery," IEEE Trans. Aerosp. Electron. Syst., vol. 26, no. 2, pp. 293-305, Mar. 1990.

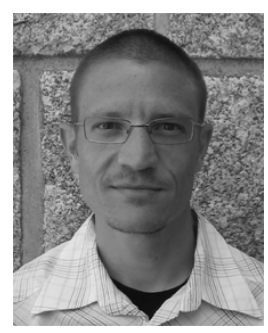

Carlos López-Martínez (S'96-M'04) received the M.Sc. degree in electrical engineering and the Ph.D. degree from the Technical University of Catalonia (UPC), Barcelona, Spain, in 1999 and 2003, respectively.

In 1999, he joined the Signal Theory and Communications Department, UPC, where he developed his $\mathrm{Ph} . \mathrm{D}$. thesis, which focused on multidimensional speckle noise modeling and reduction. From October 2000 until March 2002, he was with the Frequency and Radar Systems Department, German Aerospace Center (DLR), Oberpfaffenhofen. Since June 2003, he has been with the Image and Remote Sensing Group-SAPHIR Team, in the Institute of Electronics and Telecommunications of Rennes (IETR-CNRS UMR 6164). His research interests include SAR and multidimensional SAR, radar polarimetry, digital signal processing, estimation theory, and harmonic analysis.

Dr. López-Martínez received the Student Prize Paper Award at the EUSAR 2002 Conference, Cologne, Germany.

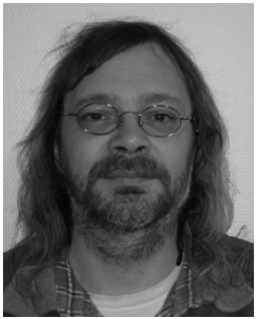

Eric Pottier (M'95) received the M.S. and Ph.D. degrees in signal processing and telecommunication from the University of Rennes 1, Rennes, France, and the Habilitation from the University of Nantes, Nantes, France, in 1987, 1990, and 1998, respectively.

From 1988 to 1999, he was an Associate Professor at IRESTE-University of Nantes, where he was the Head of the Polarimetry Group of the Electronic and Informatic Systems laboratory. Since 1999, he has been a Full Professor at the University of Rennes 1, where he is currently the Deputy Director of the Institute of Electronics and Telecommunications of Rennes (IETR-CNRS UMR 6164) and also Head of the Radar Polarimetry Remote Sensing Group. His current activities of research and education are centered in the topics of analog electronics, microwave theory, and radar imaging with emphasis in radar polarimetry. His research covers a wide spectrum of areas from radar image processing (SAR, ISAR), polarimetric scattering modeling, supervised/unsupervised polarimetric segmentation, and classification to fundamentals and basic theory of polarimetry. Since 1989, he has supervised more than 50 research students to graduation (M.Sc. and Ph.D.) in radar polarimetry covering areas from theory to remote sensing applications. He has six publications in books, 23 papers in refereed journals, and more than 160 papers in conference and symposium proceedings. He has presented advanced courses and seminars on radar polarimetry to a wide range of organizations and events (DLR, NASDA, JRC, RESTEC, ISAP2000, IGARSS03, EUSAR04, NATO, PolInSAR05, IGARSS05).

Dr. Pottier received the Best Paper Award during EUSAR2000 for his research activities, coauthored with J. S. Lee (U.S. Naval Research Laboratory), in the topic of polarimetric unsupervised segmentation of POL-SAR data. He has chaired and organized 21 sessions in International Conferences and was member of the technical and scientific committees of 17 international symposium or conferences. He has been invited to present 21 presentations in international conferences and 14 in national conferences.

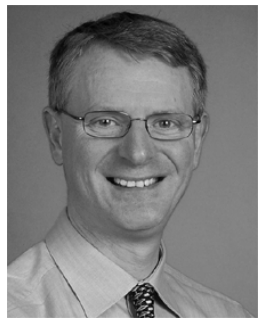

Shane R. Cloude (M'87-SM'96-F'01) was born in Chatham, England in 1959. He received the B.S. degree from the University of Dundee, Dundee, U.K., and the Ph.D. degree from the University of Birmingham, Birmingham, U.K., in 1981 and 1987, respectively, both in electrical and electronic engineering.

He was a Radar Scientist at the Royal Signals and Radar Establishment (RSRE), Great Malvern, U.K., in 1987, before taking up teaching and research positions at the University of Dundee, the University of York, and the University of Nantes. In 1996, he was the recipient of a Fellowship from the Alexander von Humboldt Foundation in Germany, working in collaboration with DLR on the development of polarimetric SAR Interferometry. Between 1997 and 2004, he was Director of Applied Electromagnetics (AELc), a research consultancy company based in Scotland, undertaking contract research in radar and antenna design for industry, government, and defense laboratories. In 2004, he was appointed to the DSTO Chair in Microwave Radar at the University of Adelaide, Adelaide, Australia. His main research interests are in the exploitation of polarization effects in radar and optical remote sensing.

Dr. Cloude is an Associate Editor of the IEEE TRANSACTIONS ON Geoscience and Remote Sensing. He is a Fellow of the Alexander von Humboldt Society in Germany and an Honorary Professor at the Universities of Dundee and York. 